ESAIM: COCV 19 (2013) 219-238

DOI: $10.1051 / \mathrm{cocv} / 2012007$
ESAIM: Control, Optimisation and Calculus of Variations

www.esaim-cocv.org

\title{
DISTRIBUTED CONTROL FOR MULTISTATE MODIFIED NAVIER-STOKES EQUATIONS
}

\author{
NADIR ARADA ${ }^{1}$
}

\begin{abstract}
The aim of this paper is to establish necessary optimality conditions for optimal control problems governed by steady, incompressible Navier-Stokes equations with shear-dependent viscosity. The main difficulty derives from the fact that equations of this type may exhibit non-uniqueness of weak solutions, and is overcome by introducing a family of approximate control problems governed by well posed generalized Stokes systems and by passing to the limit in the corresponding optimality conditions.
\end{abstract}

Mathematics Subject Classification. 49K20, 76D55, 76A05.

Received July 11, 2011. Revised November 14, 2011.

Published online May 2, 2012.

\section{INTRODUCTION}

This paper deals with an optimal control problem associated with a viscous, incompressible fluid. The controls and states are constrained to satisfy a modified Navier-Stokes system with shear dependent viscosity given by

$$
\begin{cases}-\nabla \cdot(\tau(D y))+y \cdot \nabla y+\nabla \pi=u & \text { in } \Omega \\ \nabla \cdot y=0 & \text { in } \Omega, \\ y=0 & \text { on } \Gamma\end{cases}
$$

where $y$ is the velocity field, $\pi$ is the pressure, $\tau$ is the Cauchy stress tensor, $D y=\frac{\nabla y+(\nabla y)^{T}}{2}$ is the symmetric part of the velocity gradient $\nabla y$, the control $u$ is the distributed mechanical force and $\Omega \subset \mathbb{R}^{n}(n=2$ or $n=3)$ is a bounded domain. The objective of the control is to match the velocity field to a given target field. More precisely, we consider the following optimal control problem

$$
\left(P_{\alpha}\right)\left\{\begin{array}{l}
\text { Minimize } J(y, u)=\frac{1}{2} \int_{\Omega}\left|y-y_{d}\right|^{2} \mathrm{~d} x+\frac{\nu}{2} \int_{\Omega}|u|^{2} \mathrm{~d} x \\
\text { Subject to }(y, u) \in W_{0}^{1, \alpha}(\Omega) \times U_{a d} \text { satisfies (1.1) for some } \pi \in L^{\alpha}(\Omega),
\end{array}\right.
$$

Keywords and phrases. Optimal control, multistate Navier-Stokes equations, shear-dependent viscosity, necessary optimality conditions.

1 Departamento de Matemática, Faculdade de Ciências e Tecnologia da Universidade Nova de Lisboa, Quinta da Torre, 2829-516,

Caparica, Portugal. naar@fct.unl.pt 
where $\alpha \geq 2, \nu$ is a positive constant, $y_{d}$ is some desired velocity field and $U_{a d}$, the set of admissible controls, is a nonempty convex closed subset of $L^{2}(\Omega)$.

The considered class of fluids are described by partial differential equations of the quasi-linear type that generalize the Navier-Stokes system. These equations were first studied by Ladyzhenskaya [12] and Lions [13] who proved existence of weak solutions using compactness arguments and the theory of monotone operators (see [16] for a review on the subject). Only scant attention has been paid to the analysis of optimal control problems governed by these equations. We mention the work of Slawig [18] for the two-dimensional steady case, and Wachsmuth and Roubíček [19] for the two-dimensional unsteady case. We also refer to the work of Gunzburger and Trenchea [9] devoted to optimal control problem for a three-dimensional modified NavierStokes system coupled with maxwell equations, and to the work of Kunisch and Marduel [15] for a problem with temperature-dependent viscosity.

As for optimal control problems governed by steady Navier-Stokes equations, one of the issues encountered when dealing with the class of problems studied in this paper is related with the uniqueness of the state variable, guaranteed under some restrictions on the data. Different approaches have been considered. The first one consists in deriving the necessary optimality conditions on a set of admissible controls defined by taking into account these restrictions (see e.g. [6,17]). Another method introduced by Abergel and Casas [1] allows to obtain the optimality conditions of Fritz-John type for any convex admissible control set and derive these conditions in a qualified form when the so-called $(C)$ property introduced by Gunzburger et al. in [10] is fulfilled.

Another difficulty arises when studying the differentiability of the control-to-state mapping and is a consequence of the nonlinearity of the stress tensor. Unless the gradient of the velocity is uniformly bounded, or $\alpha=2$ which corresponds to the Navier-Stokes equations, the related analysis cannot be achieved in Sobolev spaces and the natural setting for the linearized equation (and similarly for the adjoint equation) involves adequate weighted Sobolev spaces. The underlying difficulties appear identically in a class of optimal control problems governed by quasilinear elliptic equations, and we specially mention the related papers by Casas and Fernández (see $[3-5])$.

In the present work, we do not impose any additional restriction on the set of admissible controls. System (1.1) may then exhibit non-uniqueness of weak solutions and the control-to-state mapping $u \longmapsto y_{u}$ can be multivalued. Combining the methods developed in $[1,4,5]$ together with explicite estimates established in [2], we derive optimality conditions in a nonqualified form in both two-dimensional and three-dimensional cases. Their qualification is guaranteed under the $(C)$ property, or under a precise condition on the optimal control. This last result was obtained in [2] using a different approach and seems to be new in the sense that no constraint on the size of admissible controls is needed.

The plan of the paper is as follows. Assumptions, notation and some preliminary results are given in Section 2. The main results are stated in Section 3. In Section 4, we introduce a family of approximate control problems associated with well-posed generalized Stokes systems and we analyze the differentiability of the corresponding control-to-state mapping. We establish the approximate optimality conditions in Section 5 and, by passing to the limit, we derive the optimality conditions for our control problem in Section 6.

\section{Notation AND PRELIMINARY RESUlts}

Throughout the paper, $\Omega$ is a bounded domain in $\mathbb{R}^{n}(n=2$ or $n=3)$. The boundary of $\Omega$ is denoted by $\Gamma$ and is of class $C^{2}$. Since many of the quantities occuring in the paper are vector-valued functions, the notation will be abreged for the sake of brevity and we will use the same notation of norms for scalar, vector and matrix-valued functions.

For $\eta, \zeta \in \mathbb{R}^{n \times n}$, we define the scalar product and the corresponding norm by

$$
\eta: \zeta=\sum_{i, j=1}^{n} \eta_{i j} \zeta_{i j} \quad \text { and } \quad|\eta|=(\eta: \eta)^{\frac{1}{2}}
$$


For $\eta \in \mathbb{R}^{n \times n \times n \times n}$ and $\zeta \in \mathbb{R}^{n \times n}$, the scalar product is defined by

$$
\eta: \zeta=\left(\sum_{k, \ell=1}^{n} \eta_{i j k \ell} \zeta_{k \ell}\right)_{i, j=1, \ldots, n} \in \mathbb{R}^{n \times n}
$$

and we can verify that

$$
\left(\eta: \zeta_{1}\right): \zeta_{2}=\left(\zeta_{2}: \eta\right): \zeta_{1}, \quad \eta \in \mathbb{R}^{n \times n \times n \times n}, \zeta_{1}, \zeta_{2} \in \mathbb{R}^{n \times n}
$$

Let us now summarize assumptions on the nonlinear tensor $\tau$. We assume that $\tau: \mathbb{R}_{\text {sym }}^{n \times n} \longrightarrow \mathbb{R}_{\text {sym }}^{n \times n}$ has a potential, i.e. there exists a function $\Phi \in C^{2}\left(\mathbb{R}_{n}^{+}, \mathbb{R}_{n}^{+}\right)$with $\Phi(0)=0$ such that

$$
\tau_{i j}(\eta)=\frac{\partial \Phi\left(|\eta|^{2}\right)}{\partial \eta_{i j}}=2 \Phi^{\prime}\left(|\eta|^{2}\right) \eta_{i j} \quad \text { for all } \eta \in \mathbb{R}_{s y m}^{n \times n}, \quad \tau(0)=0 .
$$

(Here $\mathbb{R}_{s y m}^{n \times n}$ consists of all symetric $(n \times n)$-matrices.) Moreover, we assume that for some $\alpha \geq 2$ the following assumptions hold

A1: there exists a positive constant $\gamma$ such that for all $i, j, k, \ell=1, \ldots, n$

$$
\left|\frac{\partial \tau_{k \ell}(\eta)}{\partial \eta_{i j}}\right| \leq \gamma\left(1+|\eta|^{2}\right)^{\frac{\alpha-2}{2}} \quad \text { for all } \eta \in \mathbb{R}_{s y m}^{n \times n}
$$

A2: there exists a positive constant $\mu$ such that

$$
\tau^{\prime}(\eta): \zeta: \zeta=\sum_{i, j=1}^{n} \sum_{k, \ell=1}^{n} \frac{\partial \tau_{k \ell}(\eta)}{\partial \eta_{i j}} \zeta_{k \ell} \zeta_{i j} \geq \mu\left(1+|\eta|^{2}\right)^{\frac{\alpha-2}{2}}|\zeta|^{2} \quad \text { for all } \eta, \zeta \in \mathbb{R}_{s y m}^{n \times n}
$$

These assumptions are usually used in the literature and cover a wide range of applications in non-Newtonian fluids. Typical prototypes of extra tensors used in applications are

$$
\tau(\eta)=2 \mu\left(1+|\eta|^{2}\right)^{\frac{\alpha-2}{2}} \eta \quad \text { or } \quad \tau(\eta)=2 \mu(1+|\eta|)^{\alpha-2} \eta .
$$

We recall that a fluid is called shear thickening if $\alpha>2$ and shear thinning if $\alpha<2$. For the special case $\tau(\eta)=2 \mu \eta(\alpha=2)$, we recover the Navier-Stokes equation with viscosity coeficient $\mu>0$. Assumptions A1-A2 imply the following standard properties for the non-linear tensor $\tau$ (see [16], Chap. 5).

Continuity

$$
|\tau(\eta)| \leq \frac{n^{2} \gamma}{\alpha-1}\left(1+|\eta|^{2}\right)^{\frac{\alpha-2}{2}}|\eta|
$$

Coercivity

$$
\tau(\eta): \eta \geq \mu|\eta|^{2} \quad \text { and } \quad \tau(\eta): \eta \geq \frac{\mu}{\alpha-1}|\eta|^{\alpha} .
$$

Monotonicity

$$
\begin{aligned}
& (\tau(\eta)-\tau(\zeta)):(\eta-\zeta) \geq \mu|\eta-\zeta|^{2} \\
& (\tau(\eta)-\tau(\zeta)):(\eta-\zeta) \geq \frac{\mu}{2^{2 \alpha+1}}|\eta-\zeta|^{\alpha}
\end{aligned}
$$

where $\gamma$ and $\mu$ are the constants appearing in the assumptions A1-A2.

Let us now define some useful function spaces. The space of infinitely differentiable functions with compact support in $\Omega$ will be denoted by $\mathcal{D}(\Omega)$. The standard Sobolev spaces are denoted by $W^{k, \alpha}(\Omega)(k \in \mathbb{N}$ and 
$1<\alpha<\infty)$, and their norms by $\|\cdot\|_{k, \alpha}$. We set $W^{0, \alpha}(\Omega) \equiv L^{\alpha}(\Omega)$ and $\|\cdot\|_{\alpha} \equiv\|\cdot\|_{L^{\alpha}}$. In order to eliminate the pressure in the weak formulation of the state equation, we will work in divergence-free spaces. Consider

$$
\mathcal{V}=\{\varphi \in \mathcal{D}(\Omega) \mid \nabla \cdot \varphi=0\}
$$

and denote by $V_{\alpha}$ the closure of $\mathcal{V}$ with respect to the norm $\|\nabla \cdot\|_{\alpha}$, i.e.

$$
V_{\alpha}=\left\{\varphi \in W_{0}^{1, \alpha}(\Omega) \mid \nabla \cdot \varphi=0\right\}
$$

Following [4,5], given $y \in W_{0}^{1, \alpha}(\Omega)$, we can associate two weighted Sobolev spaces $V_{\alpha}^{y}$ and $H_{\alpha}^{y}$, where $V_{\alpha}^{y}$ is the set of functions $z \in V_{2}$ such that the norm $\|\cdot\|$ defined by

$$
\|z\|^{2}=\int_{\Omega}\left(1+|D y|^{2}\right)^{\frac{\alpha-2}{2}}|D z|^{2} \mathrm{~d} x
$$

is finite, and $H_{\alpha}^{y}$ is the completion of $\mathcal{V}$ with respect to this norm. It may be verified that $V_{\alpha}^{y}$ and $H_{\alpha}^{y}$ are Hilbert spaces and that $H_{\alpha}^{y} \subset V_{\alpha}^{y}$. Moreover, since $\alpha \geq 2$, we have

$$
V_{\alpha} \subset H_{\alpha}^{y} \subset V_{2}
$$

with continuous injections.

In the remaining part of this section, we collect some results that will be useful for the sequel. We begin by the Poincaré and the Korn inequalities and next, we point out some notable facts related with the convective term.

Lemma 2.1 (see [8], Chap. 2). Let $y$ be in $H_{0}^{1}(\Omega)$. Then the following estimate holds

$$
\|y\|_{2} \leq C_{P}\|\nabla y\|_{2} \quad \text { with } \quad C_{P}=\frac{n-1}{\sqrt{n}}|\Omega|^{\frac{1}{n}} .
$$

Lemma 2.2 (see $[11])$. Let $y$ be in $H_{0}^{1}(\Omega)$. Then we have

$$
\|\nabla y\|_{2} \leq \sqrt{2}\|D y\|_{2}
$$

with the equality if we suppose that $y \in V_{2}$.

Lemma 2.3 (see [2], Lem. 2.5). Let $y_{1}$ be in $V_{2}$ and let $y_{2}$ and $y_{3}$ be in $H_{0}^{1}(\Omega)$. Then the following estimate holds

$$
\left|\left(y_{1} \cdot \nabla y_{2}, y_{3}\right)\right| \leq \kappa_{1}\left\|D y_{1}\right\|_{2}\left\|D y_{2}\right\|_{2}\left\|D y_{3}\right\|_{2}
$$

with $\kappa_{1}=\frac{2^{\frac{3}{2}}(n-1)}{n}|\Omega|^{\frac{1}{n(n-1)}}$.

Lemma 2.4. Let $y_{1}, y_{2}$ and $y_{3}$ be in $H_{0}^{1}(\Omega)$. Then

$$
\left(y_{1} \cdot \nabla y_{2}, y_{3}\right)=-\left(y_{1} \cdot \nabla y_{3}, y_{2}\right) \quad \text { and } \quad\left(y_{1} \cdot \nabla y_{2}, y_{2}\right)=0 .
$$

Multiplying equation (1.1) by test functions $\varphi \in V_{\alpha}$ and integrating, we obtain the following weak formulation.

Definition 2.5. Let $u \in L^{2}(\Omega)$. A function $y \in V_{\alpha}$ is a weak solution of (1.1) if

$$
(\tau(D y), D \varphi)+(y \cdot \nabla y, \varphi)=(u, \varphi) \quad \text { for all } \varphi \in V_{\alpha}
$$


Let us recall that, having a solution satisfying the formulation given in Definition 2.5, it is standard to construct the corresponding pressure $\pi \in L_{0}^{\alpha}(\Omega)$ such that

$$
(\tau(D y), D \varphi)+(y \cdot \nabla y, \varphi)-(\pi, \nabla \cdot \varphi)=(u, \varphi) \quad \text { for all } \varphi \in W_{0}^{1, \alpha}(\Omega) .
$$

We will involve the pressure only in the formulations of the theorems and lemmas but not in the proofs, since it can always be reconstructed uniquely.

First mathematical investigations of (1.1) under conditions (2.1)-(2.3), were performed by Lions who proved existence of a weak solution for $\alpha \geq \frac{3 n}{n+2}$ (see [13] for more details). The restriction on the exponent $\alpha$ ensures that the convective term belongs to $L^{1}$ when considering test functions in $V_{\alpha}$. Due to Lemma 2.3, we can see that this condition is obviously satisfied when dealing with shear-thickening flows. Let us also emphasize the work by Málek et al. who established existence and regularity results for this class of problems under less restrictive assumptions (see for example $[7,14,16]$ ).

For the subsequent analysis, we state an existence and uniqueness result for the state equation and useful estimates.

Proposition 2.6 (see [2], Props. 3.3 and 3.4). Assume that $\mathbf{A}_{1}-\mathbf{A}_{2}$ are fulfilled and that $u \in L^{2}(\Omega)$. Then problem (1.1) admits a weak solution $y_{u} \in V_{\alpha}$ and the following estimates hold

$$
\left\|D y_{u}\right\|_{2} \leq \kappa_{2} \frac{\|u\|_{2}}{\mu} \quad \text { and } \quad\left\|D y_{u}\right\|_{\alpha}^{\alpha} \leq(\alpha-1)\left(\kappa_{2} \frac{\|u\|_{2}}{\mu}\right)^{2}
$$

with $\kappa_{2}=\frac{\sqrt{2}(n-1)}{\sqrt{n}}|\Omega|^{\frac{1}{n}}$. Moreover, if $u$ satisfies the following condition

$$
\frac{\|u\|_{2}}{\mu^{2}}<\frac{\sqrt{n^{3}}}{4(n-1)^{2}|\Omega|^{\frac{1}{n-1}}}
$$

then equation (1.1) admits a unique weak solution $y_{u} \in V_{\alpha}$.

Proposition 2.7 (see [2], Thm 4.1). Assume that A1-A2 are fulfilled. Then problem $\left(P_{\alpha}\right)$ admits at least a solution.

\section{Statement of the main ReSults}

In order to obtain the necessary optimality conditions for $\left(P_{\alpha}\right)$ stated in Theorem 3.1 below, a family of problems $\left(P_{\alpha}^{\varepsilon}\right)_{\varepsilon}$ whose solutions converge towards a solution of $\left(P_{\alpha}\right)$ is introduced and studied in Section 4 . We derive the optimality conditions for these approximate problems in Section 5 and we pass to the limit in these conditions in Section 6 .

Let us now formulate our main result.

Theorem 3.1. Assume that $\mathbf{A} 1-\mathbf{A} 2$ are fulfilled with $\alpha \geq 2$. Let $\bar{u} \in U_{a d}$ be a solution of $\left(P_{\alpha}\right)$ and let $\bar{y}$ be the associated state. There then exist a number $\bar{\lambda} \geq 0$ and $\bar{p} \in V_{\alpha}^{\bar{y}}$ such that the following conditions hold

$$
\begin{gathered}
\quad \bar{\lambda}+\|\bar{p}\|_{1,2} \neq 0, \\
\begin{cases}-\nabla \cdot(\tau(D \bar{y}))+\bar{y} \cdot \nabla \bar{y}+\nabla \bar{\pi}=\bar{u} & \text { in } \Omega, \\
\nabla \cdot \bar{y}=0 & \text { in } \Omega, \\
\bar{y}=0 & \text { on } \Gamma,\end{cases} \\
\begin{cases}-\nabla \cdot\left(\tau^{\prime}(D \bar{y}): D \bar{p}\right)-\bar{y} \cdot \nabla \bar{p}+(\nabla \bar{y})^{T} \bar{p}+\nabla \bar{\pi}=\bar{\lambda}\left(\bar{y}-y_{d}\right) & \text { in } \Omega, \\
\nabla \cdot \bar{p}=0 & \text { in } \Omega, \\
\bar{p}=0 & \text { on } \Gamma,\end{cases}
\end{gathered}
$$


Moreover $\bar{p}$ satisfies

$$
\left(\tau^{\prime}(D \bar{y}): D \bar{p}, D \bar{p}\right)+(\bar{p} \cdot \nabla \bar{y}, \bar{p}) \leq \bar{\lambda}\left(\bar{y}-y_{d}, \bar{p}\right) .
$$

It is obvious that these optimality conditions can be written using the weak formulations corresponding to the state and adjoint state systems. More precisely, systems (3.2) and (3.3) read as

$$
\begin{gathered}
(\tau(D \bar{y}), D \varphi)+(\bar{y} \cdot \nabla \bar{y}, \varphi)=(\bar{u}, \varphi) \quad \text { for all } \varphi \in V_{\alpha} \\
\left(\tau^{\prime}(D \bar{y}): D \varphi, D \bar{p}\right)+(\varphi \cdot \nabla \bar{y}+\bar{y} \cdot \nabla \varphi, \bar{p})=\bar{\lambda}\left(\bar{y}-y_{d}, \varphi\right) \quad \text { for all } \varphi \in H_{\alpha}^{\bar{y}} .
\end{gathered}
$$

The optimality conditions stated in Theorem 3.1 are of Fritz-John type and we are interested in the cases where $\bar{\lambda}$ can be chosen equal to one. Following Gunzburger et al. [10], the set of admissible controls $U_{a d}$ is said to have the property $(C)$ at $(\bar{y}, \bar{u})$ if for any nonzero solution $(p, \pi)$ of the system

$$
\begin{cases}-\nabla \cdot\left(\tau^{\prime}(D \bar{y}): D p\right)-\bar{y} \cdot \nabla p+(\nabla \bar{y})^{T} p+\nabla \pi=0 & \text { in } \Omega, \\ \nabla \cdot p=0 & \text { in } \Omega, \\ p=0 & \text { on } \Gamma,\end{cases}
$$

we can find $v \in U_{a d}$ such that

$$
(\bar{p}, v-\bar{u})<0 .
$$

It is obvious that if the property $(C)$ is satisfied then $\bar{\lambda} \neq 0$. Replacing $\bar{p}$ by $\frac{\bar{p}}{\lambda}$, we obtain the following result.

Corollary 3.2. Assume that assumptions of Thorem 3.1 are fulfilled. If $U_{\text {ad }}$ has the property $(C)$ at $(\bar{y}, \bar{u})$, then conditions (3.2)-(3.5) hold with $\bar{\lambda}=1$.

Another consequence of our main result is that the optimality conditions can be obtained in a qualified form if the optimal control is subject to some constraint, the same that guarantees the uniqueness of the corresponding state and adjoint state. This result seems interesting in the sense that we do not need to impose any other constraint on the admissible set of controls.

Corollary 3.3. Assume that assumptions of Thorem 3.1 are fulfilled, and assume that $\bar{u}$ satisfies

$$
\frac{\|\bar{u}\|_{2}}{\mu^{2}}<\frac{\sqrt{n^{3}}}{4(n-1)^{2}|\Omega|^{\frac{1}{n-1}}} .
$$

Then there exist a unique $\bar{y} \in V_{\alpha}$ and a unique $\bar{p} \in H_{\alpha}^{\bar{y}}$ such that conditions (3.2)-(3.4) hold with $\bar{\lambda}=1$.

Proof. Due Proposition 2.6, the state equation (3.2) admits a unique solution $\bar{y} \in V_{\alpha}$. Similarly, due Proposition 3.9 in [2], if $\bar{u}$ satisfies (3.6) then the adjoint system (3.3) admits a unique solution $\bar{p}$ in $H_{\alpha}^{\bar{y}}$. It follows that, if we suppose that $\bar{\lambda}=0$ then $\bar{p} \equiv 0$ is the (unique) solution of (3.3) leading to a contradiction with the nontriviality condition (3.1).

Notice that if the assumptions of the previous corollary are satisfied, then the solution $\bar{p}$ of (3.3) belongs to $H_{\alpha}^{\bar{y}} \subset V_{\alpha}^{\bar{y}}$, implying that inequality (3.5) is automatically satisfied. More precisely, by testing the weak formulation of (3.3) by $\bar{p}$, we obtain

$$
\left(\tau^{\prime}(D \bar{y}): D \bar{p}, D \bar{p}\right)+(\bar{p} \cdot \nabla \bar{y}, \bar{p})=\left(\bar{y}-y_{d}, \bar{p}\right) .
$$

Let us finish this section by considering the case of the Navier-Stokes equations. For $\alpha=2, V_{\alpha}^{\bar{y}} \equiv H_{\alpha}^{\bar{y}} \equiv V_{2}$ and we recover the optimality conditions already established by Abergel and Casas in [1] for a slightly different functional. 
Corollary 3.4. Assume that the extra-stress tensor has the form $\tau(\eta)=2 \mu \eta$. Let $\bar{u} \in U_{\text {ad }}$ be a solution of $\left(P_{2}\right)$ and let $\bar{y}$ be the associated state. There then exist a number $\bar{\lambda} \geq 0$ and $\bar{p} \in V_{2}$ such that the following conditions hold

$$
\begin{gathered}
\bar{\lambda}+\|\bar{p}\|_{1,2} \neq 0, \\
\begin{cases}-\mu \Delta \bar{y}+\bar{y} \cdot \nabla \bar{y}+\nabla \bar{\pi}=\bar{u} & \text { in } \Omega, \\
\nabla \cdot \bar{y}=0 & \text { in } \Omega, \\
\bar{y}=0 & \text { on } \Gamma,\end{cases} \\
\begin{cases}-\mu \Delta \bar{p}-\bar{y} \cdot \nabla \bar{p}+(\nabla \bar{y})^{T} \bar{p}+\nabla \widetilde{\pi}=\bar{\lambda}\left(\bar{y}-y_{d}\right) & \text { in } \Omega, \\
\nabla \cdot \bar{p}=0 & \text { in } \Omega, \\
\bar{p}=0 & \text { on } \Gamma,\end{cases}
\end{gathered}
$$

Moreover, if $U_{\text {ad }}$ has the property $(C)$ at $(\bar{y}, \bar{u})$ or if $\bar{u}$ satisfies (3.6), then the optimality conditions (3.2)-(3.5) hold with $\bar{\lambda}=1$.

\section{Approximate problem}

\subsection{Setting and preliminary convergence results}

Let $(\bar{u}, \bar{y})$ be a fixed solution of $\left(P_{\alpha}\right)$. Following Abergel and Casas [1], we approximate the control problem by a family of penalized problems governed by a generalized Stokes system for which uniqueness of solutions is guarenteed. More precisely, for every $\varepsilon>0$, we define a functional $J^{\varepsilon}$ by

$$
J^{\varepsilon}(w, u)=J\left(y_{w, u}, u\right)+\frac{1}{2 \varepsilon}\left\|D\left(y_{w, u}-w\right)\right\|_{2}^{2}+\frac{1}{2}\left\|y_{w, u}-\bar{y}\right\|_{2}^{2}+\frac{1}{2}\|u-\bar{u}\|_{2}^{2},
$$

where $y_{w, u}$ satisfies following (generalized) Stokes system

$$
\begin{cases}-\nabla \cdot(\tau(D y))+w \cdot \nabla y+\nabla \pi=u & \text { in } \Omega, \\ \nabla \cdot y=0 & \text { in } \Omega, \\ y=0 & \text { on } \Gamma .\end{cases}
$$

The problem $\left(P_{\alpha}^{\varepsilon}\right)$ is then defined in the following way

$$
\begin{array}{ll}
\left(P_{\alpha}^{\varepsilon}\right) \quad \text { Minimize } \quad J^{\varepsilon}(w, u) \\
\text { Subject to }(w, u) \in V_{\alpha} \times U_{a d} .
\end{array}
$$

Proposition 4.1. Let $u \in L^{2}(\Omega)$ and $w \in V_{\alpha}$. Then problem (4.1) admits a unique weak solution $y_{w, u}$ in $V_{\alpha}$. Moreover, the following estimates hold

$$
\left\|D y_{w, u}\right\|_{2} \leq \frac{\kappa_{2}}{\mu}\|u\|_{2} \quad \text { and } \quad\left\|D y_{w, u}\right\|_{\alpha}^{\alpha} \leq(\alpha-1)\left(\frac{\kappa_{2}}{\mu}\|u\|_{2}\right)^{2}
$$

with $\kappa_{2}$ defined in Proposition 2.6.

Proof. Existence and uniqueness of a solution and the corresponding estimates can be established by using standard arguments (see for example Props. 3.3. and 3.4 in [2]). 
We next prove that each problem $\left(P_{\alpha}^{\varepsilon}\right)$ admits at least a solution $\left(w^{\varepsilon}, u^{\varepsilon}\right)$ and that these solutions form an approximating family for $\left(P_{\alpha}\right)$

Proposition 4.2. For every $\varepsilon>0$ there exists at least one solution $\left(w^{\varepsilon}, u^{\varepsilon}\right)$ of $\left(P_{\alpha}^{\varepsilon}\right)$. Moreover, if we denote by $y^{\varepsilon}$ the solution of (4.1) corresponding to $\left(w^{\varepsilon}, u^{\varepsilon}\right)$, then we have

$$
\begin{gathered}
\lim _{\varepsilon \rightarrow 0}\left\|u^{\varepsilon}-\bar{u}\right\|_{2}=\lim _{\varepsilon \rightarrow 0} \frac{1}{\varepsilon}\left\|D\left(y^{\varepsilon}-w^{\varepsilon}\right)\right\|_{2}=0, \\
\lim _{\varepsilon \rightarrow 0}\left\|D\left(w^{\varepsilon}-\bar{y}\right)\right\|_{2}=\lim _{\varepsilon \rightarrow 0}\left\|D\left(y^{\varepsilon}-\bar{y}\right)\right\|_{\alpha}=0, \\
\lim _{\varepsilon \rightarrow 0} J^{\varepsilon}\left(w^{\varepsilon}, u^{\varepsilon}\right)=J(\bar{y}, \bar{u}) .
\end{gathered}
$$

Proof. Existence of an optimal solution $\left(w^{\varepsilon}, u^{\varepsilon}\right)$ of $\left(P_{\alpha}^{\varepsilon}\right)$ is standard and can be proved by using standard arguments. Since $y_{\bar{y}, \bar{u}}=\bar{y}$, we have

$$
J^{\varepsilon}\left(w^{\varepsilon}, u^{\varepsilon}\right) \leq J^{\varepsilon}(\bar{y}, \bar{u})=J(\bar{y}, \bar{u})
$$

implying that

$$
\left\|u^{\varepsilon}\right\|_{2}^{2} \leq 2\left\|u^{\varepsilon}-\bar{u}\right\|_{2}^{2}+2\|\bar{u}\|_{2}^{2} \leq 4 J(\bar{y}, \bar{u})+2\|\bar{u}\|_{2}^{2},
$$

and

$$
\left\|D\left(y^{\varepsilon}-w^{\varepsilon}\right)\right\|_{2}^{2} \leq 2 \varepsilon J(\bar{y}, \bar{u}) \longrightarrow 0 \quad \text { when } \varepsilon \rightarrow 0 .
$$

Setting $\varphi=y^{\varepsilon}$ in the weak formulation of (4.1) yields

$$
\left(\tau\left(D y^{\varepsilon}\right), D y^{\varepsilon}\right)=\left(u^{\varepsilon}, y^{\varepsilon}\right) .
$$

Taking into account (2.2), the Poincaré and the Korn inequalities, we obtain

$$
\mu\left\|D y^{\varepsilon}\right\|_{2}^{2} \leq\left\|u^{\varepsilon}\right\|_{2}\left\|y^{\varepsilon}\right\|_{2} \leq \kappa_{2}\left\|u^{\varepsilon}\right\|_{2}\left\|D y^{\varepsilon}\right\|_{2}
$$

which together with (4.2) give

$$
\left\|D y^{\varepsilon}\right\|_{2} \leq \frac{\kappa_{2}}{\mu}\left\|u^{\varepsilon}\right\|_{2} \leq \frac{\kappa_{2}}{\mu}\left(4 J(\bar{y}, \bar{u})+2\|\bar{u}\|_{2}^{2}\right)^{\frac{1}{2}}
$$

and

$$
\mu\left\|D y^{\varepsilon}\right\|_{\alpha}^{\alpha} \leq\left\|u^{\varepsilon}\right\|_{2}\left\|y^{\varepsilon}\right\|_{2} \leq \kappa_{2}\left\|u^{\varepsilon}\right\|_{2}\left\|D y^{\varepsilon}\right\|_{2} \leq \frac{\kappa_{2}^{2}}{\mu}\left(4 J(\bar{y}, \bar{u})+2\|\bar{u}\|_{2}^{2}\right) .
$$

The previous estimate together with (2.1) imply

$$
\begin{aligned}
\left\|\tau\left(D y^{\varepsilon}\right)\right\|_{\alpha^{\prime}}^{\alpha^{\prime}} & \leq\left(\frac{n^{2} \gamma}{\alpha-1}\right)^{\alpha^{\prime}} \int_{\Omega}\left(1+\left|D y^{\varepsilon}\right|^{2}\right)^{\frac{\alpha-2}{2} \alpha^{\prime}}\left|D y^{\varepsilon}\right|^{\alpha^{\prime}} \mathrm{d} x \\
& \leq\left(\frac{n^{2} \gamma}{\alpha-1}\right)^{\alpha^{\prime}} \int_{\Omega}\left(1+\left|D y^{\varepsilon}\right|^{2}\right)^{\frac{\alpha}{2}} \mathrm{~d} x \leq\left(\frac{n^{2} \gamma}{\alpha-1}\right)^{\alpha^{\prime}} 2^{\frac{\alpha-2}{2}}\left(|\Omega|+\left\|D y^{\varepsilon}\right\|_{\alpha}^{\alpha}\right) \\
& \leq\left(\frac{n^{2} \gamma}{\alpha-1}\right)^{\alpha^{\prime}} 2^{\frac{\alpha-2}{2}}\left(|\Omega|+\left(\frac{\kappa_{2}}{\mu}\right)^{2}\left(4 J(\bar{y}, \bar{u})+2\|\bar{u}\|_{2}^{2}\right)\right)
\end{aligned}
$$

and the sequence $\left(\tau\left(D y^{\varepsilon}\right)\right)_{\varepsilon}$ is uniformly bounded in $L^{\alpha^{\prime}}(\Omega)$. There then exist subsequences, still indexed by $\varepsilon$, $\left(u^{\varepsilon}\right)_{\varepsilon},\left(y^{\varepsilon}\right)_{\varepsilon},\left(w^{\varepsilon}\right)_{\varepsilon}$ and $\left(\tau\left(D y^{\varepsilon}\right)\right)_{\varepsilon}$, and $(u, y, \widetilde{\tau}) \in L^{2}(\Omega) \times V_{\alpha} \times L^{\alpha^{\prime}}(\Omega)$ such that $\left(u^{\varepsilon}\right)_{\varepsilon}$ weakly converges to $u$ in $L^{2}(\Omega),\left(y^{\varepsilon}\right)_{\varepsilon}$ and $\left(w^{\varepsilon}\right)_{\varepsilon}$ weakly converge to $y$ in $V_{\alpha}$, and $\left(\tau\left(D y^{\varepsilon}\right)\right)_{\varepsilon}$ weakly converges to $\widetilde{\tau}$ in $L^{\alpha^{\prime}}(\Omega)$. Moreover, 
since $\alpha \geq 2>\frac{3 n}{n+2}$, by using compactness results on Sobolev spaces, we deduce that $\left(w^{\varepsilon}\right)_{\varepsilon}$ strongly converges to $y$ in $L^{\frac{2 \alpha}{\alpha-1}}(\Omega)$ and for all $\varphi \in V_{\alpha}$, we have

$$
\begin{aligned}
\left|\left(w^{\varepsilon} \cdot \nabla w^{\varepsilon}-y \cdot \nabla y, \varphi\right)\right| & \leq\left|\left(\left(w^{\varepsilon}-y\right) \cdot \nabla w^{\varepsilon}, \varphi\right)\right|+\left|\left(y \cdot \nabla\left(w^{\varepsilon}-y\right), \varphi\right)\right| \\
& =\left|\left(\left(w^{\varepsilon}-y\right) \cdot \nabla w^{\varepsilon}, \varphi\right)\right|+\left|\left(y \cdot \nabla \varphi, w^{\varepsilon}-y\right)\right| \\
& \leq\left(\left\|\nabla w^{\varepsilon}\right\|_{\alpha}\|\varphi\|_{\frac{2 \alpha}{\alpha-1}}+\|y\|_{\frac{2 \alpha}{\alpha-1}}\|\nabla \varphi\|_{\alpha}\right)\left\|w^{\varepsilon}-y\right\|_{\frac{2 \alpha}{\alpha-1}} \\
& \longrightarrow 0 \quad \text { when } \varepsilon \rightarrow 0 .
\end{aligned}
$$

Taking into account these convergence results, by passing to the limit in the weak formulation corresponding to $y^{\varepsilon}$ and using classical arguments, we obtain

$$
(\tau(D y), D \varphi)+(y \cdot \nabla y, \varphi)=(u, \varphi) \quad \text { for all } \varphi \in V_{\alpha} .
$$

Therefore $y$ is the solution of $(1.1)$ corresponding to $u$ and $(y, u)$ is admissible for $\left(P_{\alpha}\right)$. To prove that $\left(y^{\varepsilon}\right)_{\varepsilon>0}$ strongly converges to $y$ in $W_{0}^{1, \alpha}(\Omega)$, notice that due to the monotonicity condition $(2.3)_{2}$, Lemma 2.4 and classical embedding results, we have

$$
\begin{aligned}
& \frac{\mu}{2^{2 \alpha+1}\left\|D\left(y^{\varepsilon}-y\right)\right\|_{\alpha}^{\alpha}} \leq\left(\tau\left(D y^{\varepsilon}\right)-\tau(D y), D\left(y^{\varepsilon}-y\right)\right) \\
&=\left(u^{\varepsilon}-u, y^{\varepsilon}-y\right)+\left(y \cdot \nabla y-w^{\varepsilon} \cdot \nabla y^{\varepsilon}, y^{\varepsilon}-y\right) \\
&=\left(u^{\varepsilon}-u, y^{\varepsilon}-y\right)+\left(\left(y-w^{\varepsilon}\right) \cdot \nabla y, y^{\varepsilon}-y\right) \\
& \longrightarrow 0 \quad \text { when } \varepsilon \rightarrow 0 .
\end{aligned}
$$

Finally, since $(\bar{y}, \bar{u})$ is a solution of $\left(P_{\alpha}\right)$, we have

$$
\begin{aligned}
J(y, u) & \leq J(y, u)+\frac{1}{2}\|y-\bar{y}\|_{2}^{2} \mathrm{~d} x+\frac{1}{2}\|u-\bar{u}\|_{2}^{2} \\
& \leq \liminf _{\varepsilon \rightarrow 0} J^{\varepsilon}\left(w^{\varepsilon}, u^{\varepsilon}\right) \leq J(\bar{y}, \bar{u}) \leq J(y, u) .
\end{aligned}
$$

and consequently $y \equiv \bar{y}$ and $u \equiv \bar{u}$. On the other hand

$$
J(\bar{y}, \bar{u}) \leq \liminf _{\varepsilon \rightarrow 0} J^{\varepsilon}\left(w^{\varepsilon}, u^{\varepsilon}\right) \leq \limsup _{\varepsilon \rightarrow 0} J^{\varepsilon}\left(w^{\varepsilon}, u^{\varepsilon}\right) \leq J(\bar{y}, \bar{u})
$$

and thus

$$
\begin{aligned}
\limsup _{\varepsilon \rightarrow 0}\left(\frac{1}{2 \varepsilon}\left\|D\left(y^{\varepsilon}-w^{\varepsilon}\right)\right\|_{\alpha}^{\alpha} \mathrm{d} x+\frac{1}{2}\left\|u^{\varepsilon}-\bar{u}\right\|_{2}\right) & \leq \limsup _{\varepsilon \rightarrow 0}\left(J^{\varepsilon}\left(w^{\varepsilon}, u^{\varepsilon}\right)-J\left(y^{\varepsilon}, u^{\varepsilon}\right)\right) \\
& \leq \limsup _{\varepsilon \rightarrow 0} J^{\varepsilon}\left(w^{\varepsilon}, u^{\varepsilon}\right)-\liminf _{\varepsilon \rightarrow 0} J\left(y^{\varepsilon}, u^{\varepsilon}\right) \leq 0 .
\end{aligned}
$$

The claimed result is then proven.

In order to establish the approximate optimality conditions corresponding to $\left(P_{\alpha}^{\varepsilon}\right)$, we need to study the properties of the corresponding control-to-state mapping. Consider

$$
\begin{aligned}
G: V_{\alpha} \times L^{2}(\Omega) & \longrightarrow V_{2} \\
(w, u) & \longmapsto y_{w, u}
\end{aligned}
$$

where $y_{w, u}$ is the solution of (4.1). In the next section, we derive some estimates necessary to prove the Lipschitz continuity of $G$. Existence and uniqueness results for an auxiliary linearized system are established in Section 4.3 while Section 4.4 is devoted to the the analysis of the differentiability of $G$. 


\subsection{Lipschitz continuity of the control-to-state mapping}

Lemma 4.3. For $i=1,2$, let $\left(u_{i}, w_{i}\right)$ be in $L^{2}(\Omega) \times V_{\alpha}$ and let $y_{w_{i}, u_{i}}$ be the corresponding weak solution of (4.1). Then the following estimates hold

$$
\begin{aligned}
\left\|D\left(y_{w_{1}, u_{1}}-y_{w_{2}, u_{2}}\right)\right\|_{2} & \leq \frac{\kappa_{2}}{\mu}\left(\left\|u_{1}-u_{2}\right\|_{2}+\frac{\kappa_{1}\left\|u_{1}\right\|_{2}}{\mu}\left\|D\left(w_{1}-w_{2}\right)\right\|_{2}\right) \\
\left\|D\left(y_{w_{1}, u_{1}}-y_{w_{2}, u_{2}}\right)\right\|_{\alpha}^{\alpha} & \leq 2^{2 \alpha+1}\left(\frac{\kappa_{2}}{\mu}\left(\left\|u_{1}-u_{2}\right\|_{2}+\frac{\kappa_{1}\left\|u_{1}\right\|_{2}}{\mu}\left\|D\left(w_{2}-w_{1}\right)\right\|_{2}\right)\right)^{2}
\end{aligned}
$$

with $\kappa_{1}$ and $\kappa_{2}$ respectively defined in Lemma 2.3 and Proposition 2.6.

Proof. To simplify the notation, set $y_{i}=y_{w_{i}, u_{i}}$. Substituing in the weak formulation of (4.1), setting $\varphi=y_{1}-y_{2}$ and using Lemma 2.4, we get

$$
\begin{aligned}
\left(\tau\left(D y_{1}\right)-\tau\left(D y_{2}\right), D\left(y_{1}-y_{2}\right)\right) & =\left(u_{1}-u_{2}, y_{1}-y_{2}\right)+\left(w_{2} \cdot \nabla y_{2}-w_{1} \cdot \nabla y_{1}, y_{1}-y_{2}\right) \\
& =\left(u_{1}-u_{2}, y_{1}-y_{2}\right)+\left(\left(w_{2}-w_{1}\right) \cdot \nabla y_{1}, y_{1}-y_{2}\right)-\left(w_{2} \cdot \nabla\left(y_{1}-y_{2}\right), y_{1}-y_{2}\right) \\
& =\left(u_{1}-u_{2}, y_{1}-y_{2}\right)+\left(\left(w_{2}-w_{1}\right) \cdot \nabla y_{1}, y_{1}-y_{2}\right) .
\end{aligned}
$$

Using the Poincaré and the Korn inequalities, we obtain

$$
\left|\left(u_{1}-u_{2}, y_{1}-y_{2}\right)\right| \leq\left\|u_{1}-u_{2}\right\|_{2}\left\|y_{1}-y_{2}\right\|_{2} \leq \kappa_{2}\left\|u_{1}-u_{2}\right\|_{2}\left\|D\left(y_{1}-y_{2}\right)\right\|_{2} .
$$

On the other hand, due to Lemma 2.3 and Proposition 4.1, we have

$$
\begin{aligned}
\left|\left(\left(w_{2}-w_{1}\right) \cdot \nabla y_{1}, y_{1}-y_{2}\right)\right| & \leq \kappa_{1}\left\|D\left(w_{2}-w_{1}\right)\right\|_{2}\left\|D y_{1}\right\|_{2}\left\|D\left(y_{1}-y_{2}\right)\right\|_{2} \\
& \leq \kappa_{1} \frac{\kappa_{2}\left\|u_{1}\right\|_{2}}{\mu}\left\|D\left(w_{2}-w_{1}\right)\right\|_{2}\left\|D\left(y_{1}-y_{2}\right)\right\|_{2} .
\end{aligned}
$$

By combining these estimates and taking into account the monotonicity condition $(2.3)_{1}$, we deduce that

$$
\mu\left\|D\left(y_{1}-y_{2}\right)\right\|_{2}^{2} \leq\left(\tau\left(D y_{1}\right)-\tau\left(D y_{2}\right), D\left(y_{1}-y_{2}\right)\right) \leq \kappa_{2}\left(\left\|u_{1}-u_{2}\right\|_{2}+\frac{\kappa_{1}\left\|u_{1}\right\|_{2}}{\mu}\left\|D\left(w_{2}-w_{1}\right)\right\|_{2}\right)\left\|D\left(y_{1}-y_{2}\right)\right\|_{2}
$$

implying the first estimate. Due to $(2.3)_{2}$ and (4.5), it follows that

$$
\begin{aligned}
2^{-(2 \alpha+1)} \mu\left\|D\left(y_{1}-y_{2}\right)\right\|_{\alpha}^{\alpha} & \leq\left(\tau\left(D y_{1}\right)-\tau\left(D y_{2}\right), D\left(y_{1}-y_{2}\right)\right) \\
& \leq \kappa_{2}\left(\left\|u_{1}-u_{2}\right\|_{2}+\frac{\kappa_{1}\left\|u_{1}\right\|_{2}}{\mu}\left\|D\left(w_{2}-w_{1}\right)\right\|_{2}\right)\left\|D\left(y_{1}-y_{2}\right)\right\|_{2} \\
& \leq \frac{\kappa_{2}^{2}}{\mu}\left(\left\|u_{1}-u_{2}\right\|_{2}+\frac{\kappa_{1}\left\|u_{1}\right\|_{2}}{\mu}\left\|D\left(w_{2}-w_{1}\right)\right\|_{2}\right)^{2}
\end{aligned}
$$

which gives the second estimate and completes the proof.

Lemma 4.4. For $i=1,2$, let $\left(u_{i}, w_{i}\right)$ be in $L^{2}(\Omega) \times V_{\alpha}$ and let $y_{w_{i}, u_{i}}$ be the corresponding weak solution of (4.1). Then the following estimate holds

$$
\left\|y_{w_{1}, u_{1}}-y_{w_{2}, u_{2}}\right\|_{H_{\alpha}^{y w_{2}, u_{2}}} \leq \frac{\kappa_{2} \kappa_{3}}{\mu}\left(\left\|u_{1}-u_{2}\right\|_{2}+\frac{\kappa_{1}\left\|u_{1}\right\|_{2}}{\mu}\left\|D\left(w_{2}-w_{1}\right)\right\|_{2}\right)
$$

with $\kappa_{3}=2^{\frac{\alpha-2}{2}}\left(1+2^{2 \alpha+1}\right)^{\frac{1}{2}}$. 
Proof. By taking into account assumption A2 and using standard arguments, for every $\eta, \zeta \in \mathbb{R}_{s y m}^{n \times n}$ we have

$$
\begin{aligned}
(\tau(\eta)-\tau(\zeta)):(\eta-\zeta) & =\sum_{i, j}\left(\tau_{i j}(\eta)-\tau_{i j}(\zeta)\right)(\eta-\zeta)_{i j} \\
& =\sum_{i, j=1}^{n} \int_{0}^{1} \frac{\mathrm{d}}{\mathrm{d} s} \tau_{i j}(\zeta+s(\eta-\zeta)) \mathrm{d} s(\eta-\zeta)_{i j} \\
& =\sum_{i, j=1}^{n} \sum_{k, \ell=1}^{n} \int_{0}^{1} \frac{\partial \tau_{i j}}{\partial \eta_{k \ell}}(\zeta+s(\eta-\zeta))(\eta-\zeta)_{k \ell} \mathrm{d} s(\eta-\zeta)_{i j} \\
& =\int_{0}^{1} \tau^{\prime}(\zeta+s(\eta-\zeta)):(\eta-\zeta):(\eta-\zeta) \mathrm{d} s \\
& \geq \mu \int_{0}^{1}\left(1+|\zeta+s(\eta-\zeta)|^{2}\right)^{\frac{\alpha-2}{2}}|\eta-\zeta|^{2} \mathrm{~d} s \\
& =\mu\left(1+\left|\zeta+s^{*}(\eta-\zeta)\right|^{2}\right)^{\frac{\alpha-2}{2}}|\eta-\zeta|^{2}
\end{aligned}
$$

where $0<s^{*}<1$ is a number arising when applying the mean values thorem to the integral. On the other hand, for every $s \in[0,1]$, we have

$$
\begin{aligned}
\left(1+|\zeta|^{2}\right)^{\frac{\alpha-2}{2}} & \leq\left(1+2|\zeta+s(\eta-\zeta)|^{2}+2|\eta-\zeta|^{2}\right)^{\frac{\alpha-2}{2}} \\
& \leq 2^{\alpha-2}\left(\left(1+|\zeta+s(\eta-\zeta)|^{2}\right)^{\frac{\alpha-2}{2}}+|\eta-\zeta|^{\alpha-2}\right) .
\end{aligned}
$$

Combining (4.6) and (4.7), we obtain

$$
\begin{aligned}
2^{2-\alpha}\left(1+|\zeta|^{2}\right)^{\frac{\alpha-2}{2}}|\zeta-\eta|^{2} & \leq\left(1+\left|\zeta+s^{*}(\eta-\zeta)\right|^{2}\right)^{\frac{\alpha-2}{2}}|\eta-\zeta|^{2}+|\eta-\zeta|^{\alpha} \\
& \leq \frac{1}{\mu}(\tau(\eta)-\tau(\zeta)):(\eta-\zeta)+|\eta-\zeta|^{\alpha}
\end{aligned}
$$

and thus

$$
\begin{aligned}
2^{2-\alpha}\left\|\left(1+\left|D y_{w_{2}, u_{2}}\right|^{2}\right)^{\frac{\alpha-2}{4}} D\left(y_{w_{1}, u_{1}}-y_{w_{2}, u_{2}}\right)\right\|_{2}^{2} \leq & \frac{1}{\mu}\left(\tau\left(D y_{w_{1}, u_{1}}\right)-\tau\left(D y_{w_{2}, u_{2}}\right), D\left(y_{w_{1}, u_{1}}-y_{w_{2}, u_{2}}\right)\right) \\
& +\left\|D\left(y_{w_{1}, u_{1}}-y_{w_{2}, u_{2}}\right)\right\|_{\alpha}^{\alpha} .
\end{aligned}
$$

The conclusion is a direct consequence of inequality (4.5) and Lemma 4.3.

\subsection{Linearized equation}

To show the differentiability of $G$, we need to investigate the following linearized system

$$
\begin{cases}-\nabla \cdot\left(\tau^{\prime}(D y): D z\right)+w \cdot \nabla z+\nabla \pi=v-f \cdot \nabla y & \text { in } \Omega, \\ \nabla \cdot z=0 & \text { in } \Omega, \\ z=0 & \text { on } \Gamma,\end{cases}
$$

where $v \in L^{2}(\Omega)$ and $(f, w, y) \in\left(V_{\alpha}\right)^{3}$.

Definition 4.5. A function $z$ is a weak solution of (4.8) if

$$
\left(\tau^{\prime}(D y): D z, D \varphi\right)+(w \cdot \nabla z, \varphi)=(v-f \cdot \nabla y, \varphi) \quad \text { for all } \varphi \in H_{\alpha}^{y} .
$$


Proposition 4.6. Let $v \in L^{2}(\Omega)$ and $(f, w, y) \in\left(V_{\alpha}\right)^{3}$. Then problem (4.8) admits a unique solution $z$ in $H_{\alpha}^{y}$. Moreover, the following estimate holds

$$
\|z\|_{H_{\alpha}^{y}} \leq \frac{1}{\mu}\left(\kappa_{2}\|v\|_{2}+\kappa_{1}\|D f\|_{2}\|D y\|_{2}\right) .
$$

Proof. Consider the bilinear form defined by

$$
B\left(z_{1}, z_{2}\right)=\left(\tau^{\prime}(D y): D z_{1}, D z_{2}\right)+\left(w \cdot \nabla z_{1}, z_{2}\right) .
$$

Let us first prove that $B$ is coercive on $H_{\alpha}^{y}$. Taking into account Lemma 2.4 and assumption A2, we have

$$
\begin{aligned}
B(z, z) & =\left(\tau^{\prime}(D y): D z, D z\right)+(w \cdot \nabla z, z)=\left(\tau^{\prime}(D y): D z, D z\right) \\
& \geq \nu \int_{\Omega}\left(1+|D y|^{2}\right)^{\frac{\alpha}{2}-1}|D z|^{2} \mathrm{~d} x=\nu\|z\|_{H_{\alpha}^{y}}^{2},
\end{aligned}
$$

for every $z \in H_{\alpha}^{y}$. On the other hand, Lemma 2.3 and assumption A1 yield

$$
\begin{aligned}
\left|\left(\tau^{\prime}(D y): D z_{1}, D z_{2}\right)\right| & \leq \gamma \int_{\Omega}\left(1+|D y|^{2}\right)^{\frac{\alpha-2}{2}}\left|D z_{1} \| D z_{2}\right| \mathrm{d} x \\
& \leq \gamma\left\|\left(1+|D y|^{2}\right)^{\frac{\alpha-2}{4}} D z_{1}\right\|_{2}\left\|\left(1+|D y|^{2}\right)^{\frac{\alpha-2}{4}} D z_{2}\right\|_{2}=\gamma\left\|z_{1}\right\|_{H_{\alpha}^{y}}\left\|z_{2}\right\|_{H_{\alpha}^{y}}
\end{aligned}
$$

and

$$
\left|\left(w \cdot \nabla z_{1}, z_{2}\right)\right| \leq \kappa_{2}\|D w\|_{2}\left\|D z_{1}\right\|_{2}\left\|D z_{2}\right\|_{2} \leq \kappa_{2}\|D w\|_{2}\left\|z_{1}\right\|_{H_{\alpha}^{y}}\left\|z_{2}\right\|_{H_{\alpha}^{y}}
$$

for every $z_{1}, z_{2} \in H_{\alpha}^{y_{u}}$. Therefore,

$$
B\left(z_{1}, z_{2}\right) \leq\left(\gamma+\kappa_{2}\|D w\|_{2}\right)\left\|z_{1}\right\|_{H_{\alpha}^{y}}\left\|z_{2}\right\|_{H_{\alpha}^{y}} .
$$

The bilinear form $B$ is then continuous and coercive on $H_{\alpha}^{y}$. Applying the Lax-Milgram theorem, we deduce that problem (4.8) admits a unique solution $z$ in $H_{\alpha}^{y}$. Taking into account (4.9) and Lemma 2.3, we obtain

$$
\begin{aligned}
\mu\|z\|_{H_{\alpha}^{y}}^{2} & \leq B(z, z)=(u, z)-(f \cdot \nabla y, z) \\
& \leq\|v\|_{2}\|z\|_{2}+|(f \cdot \nabla y, z)| \leq\left(\kappa_{2}\|u\|_{2}+\kappa_{1}\|D f\|_{2}\|D y\|_{2}\right)\|D z\|_{2} \\
& \leq\left(\kappa_{2}\|u\|_{2}+\kappa_{1}\|D f\|_{2}\|D y\|_{2}\right)\|z\|_{H_{\alpha}^{y}}
\end{aligned}
$$

which gives the estimate.

\subsection{Differentiability of the control-to-state mapping}

As referred in the introduction, the ideas of the proof dealing with the nonlinear tensor when studying the Gâteaux diferentiability of the control-to-state mapping are mainly due to Casas and Fernández, and were developed in $[4,5]$ to study optimal control problems governed by quasi-linear elliptic equations. By taking into account the corresponding estimates and managing the convective term, these arguments are adapted to our specific problem in Lemma 4.9 and Proposition 4.11 below, and the proofs are given for the confort of the reader.

For $u$ and $v$ in $L^{2}(\Omega), w$ and $f$ in $V_{\alpha}$ and $\rho$ in $] 0,1\left[\right.$, set $u_{\rho}=u+\rho v, w_{\rho}=w+\rho f$, and let $y_{w_{\rho}, u_{\rho}}$ and $y_{w, u}$ be the solution of (4.1) corresponding to $\left(w_{\rho}, u_{\rho}\right)$ and $(w, u)$ respectively. In order to simplify the notation we set $y_{\rho}$ instead of $y_{w_{\rho}, u_{\rho}}, y$ instead of $y_{w, u}$ and $z_{\rho}=\frac{y_{\rho}-y}{\rho}$, throughout this section. Substituing in the weak formulation of (4.1), we obtain

$$
\left(\tau\left(D y_{\rho}\right)-\tau(D y), D \varphi\right)+\left(w_{\rho} \cdot \nabla y_{\rho}-w \cdot \nabla y, \varphi\right)=\rho(v, \varphi) \quad \text { for all } \varphi \in V_{\alpha} .
$$


Lemma 4.7. The following estimates hold

$$
\begin{aligned}
\left\|D z_{\rho}\right\|_{2} & \leq \frac{\kappa_{2}}{\mu}\left(\|v\|_{2}+\frac{\kappa_{1}}{\mu}\left\|u_{\rho}\right\|_{2}\|D f\|_{2}\right) \\
\left\|D z_{\rho}\right\|_{\alpha}^{\alpha} & \leq 2^{2 \alpha+1} \rho^{2-\alpha}\left(\frac{\kappa_{2}}{\mu}\left(\|v\|_{2}+\frac{\kappa_{1}}{\mu}\left\|u_{\rho}\right\|_{2}\|D f\|_{2}\right)\right)^{2} \\
\left\|z_{\rho}\right\|_{H_{\alpha}^{y}} & \leq \frac{\kappa_{3} \kappa_{2}}{\mu}\left(\|v\|_{2}+\frac{\kappa_{1}}{\mu}\left\|u_{\rho}\right\|_{2}\|D f\|_{2}\right) .
\end{aligned}
$$

Proof. The first and second estimates are direct consequence of Lemma 4.3. The third estimate follows from Lemma 4.4.

Lemma 4.8. If $\left(z_{\rho_{k}}\right)_{k}$ weakly converges to $z$ in $V_{2}$ for some sequence $\left(\rho_{k}\right)_{k}$ converging to zero, then

$$
\lim _{k \rightarrow+\infty} \frac{1}{\rho_{k}}\left(w_{\rho_{k}} \cdot \nabla y_{\rho_{k}}-w \cdot \nabla y, \varphi\right)=(w \cdot \nabla z+f \cdot \nabla y, \varphi) \quad \text { for all } \varphi \in V_{2} .
$$

Proof. Notice that

$$
\begin{aligned}
\left|\frac{1}{\rho_{k}}\left(w_{\rho_{k}} \cdot \nabla y_{\rho_{k}}-w \cdot \nabla y, \varphi\right)-(w \cdot \nabla z+f \cdot \nabla y, \varphi)\right| & =\left|\left(f \cdot \nabla y_{\rho_{k}}, \varphi\right)+\left(w \cdot \nabla z_{\rho_{k}}, \varphi\right)-(w \cdot \nabla z+f \cdot \nabla y, \varphi)\right| \\
& =\left|\left(f \cdot \nabla\left(y_{\rho_{k}}-y\right), \varphi\right)+\left(w \cdot \nabla\left(z_{\rho_{k}}-z\right), \varphi\right)\right| \\
& =\left|\left(f \cdot \nabla\left(y_{\rho_{k}}-y\right), \varphi\right)-\left(w \cdot \nabla \varphi, z_{\rho_{k}}-z\right)\right| \\
& \leq\|f\|_{4}\left\|\nabla\left(y_{\rho_{k}}-y\right)\right\|_{2}\|\varphi\|_{4}+\|w\|_{4}\|\nabla \varphi\|_{2}\left\|z_{\rho_{k}}-z\right\|_{4} .
\end{aligned}
$$

The result is then a consequence of the strong convergence of $\left(y_{\rho_{k}}\right)_{k}$ to $y$ in $H_{0}^{1}(\Omega)$ and the strong convergence of $\left(z_{\rho_{k}}\right)_{k}$ to $z$ in $L^{4}(\Omega)$.

Lemma 4.9. If $\left(z_{\rho_{k}}\right)_{k}$ weakly converges to $z$ in $H_{\alpha}^{y}$ for some sequence $\left(\rho_{k}\right)_{k}$ converging to zero, then

$$
\lim _{k \rightarrow+\infty} \frac{1}{\rho_{k}}\left(\tau\left(D y_{\rho_{k}}\right)-\tau(D y), D \varphi\right)=\left(\tau^{\prime}(D y): D z, D \varphi\right) \quad \text { for all } \varphi \in \mathcal{V}
$$

Proof. Let $\varphi \in \mathcal{V}$ be fixed. Standard calculation shows that

$$
\begin{aligned}
\frac{1}{\rho_{k}}\left(\tau\left(D y_{\rho_{k}}\right)-\tau(D y), D \varphi\right) & =\int_{\Omega} \int_{0}^{1} \tau^{\prime}\left(D y(x)+s D\left(y_{\rho_{k}}-y\right)(x)\right): D z_{\rho_{k}}(x): D \varphi(x) \mathrm{d} s \mathrm{~d} x \\
& =\int_{\Omega} \tau^{\prime}\left(\sigma_{\rho_{k}}^{\varphi}(x)\right): D z_{\rho_{k}}(x): D \varphi(x) \mathrm{d} x=\left(\tau^{\prime}\left(\sigma_{\rho_{k}}^{\varphi}\right): D z_{\rho_{k}}, D \varphi\right),
\end{aligned}
$$

where $\sigma_{\rho_{k}}^{\varphi}(x)=D y(x)+s_{\rho_{k}}^{\varphi}(x) D\left(y_{\rho_{k}}-y\right)(x)$ with $0<s_{\rho_{k}}^{\varphi}(x)<1$ being a number (depending on $\left.\varphi(x)\right)$ arising when applying the mean values theorem to the integral in the interval $[0,1]$. Since $\alpha \geq 2$, we have

$$
\begin{aligned}
\left(1+\left|\sigma_{\rho_{k}}^{\varphi}(x)\right|^{2}\right)^{\frac{\alpha-2}{2}} & =\left(1+\left|D y(x)+s_{\rho_{k}}^{\varphi}(x) D\left(y_{\rho_{k}}-y\right)(x)\right|^{2}\right)^{\frac{\alpha-2}{2}} \\
& \leq\left(1+2|D y(x)|^{2}+2\left(s_{\rho_{k}}^{\varphi}(x)\right)^{2}\left|D\left(y_{\rho_{k}}-y\right)(x)\right|^{2}\right)^{\frac{\alpha-2}{2}} \\
& \leq 2^{\frac{\alpha-2}{2}}\left(1+|D y(x)|^{2}+\left|D\left(y_{\rho_{k}}-y\right)(x)\right|^{2}\right)^{\frac{\alpha-2}{2}} \\
& \leq 2^{\alpha-2}\left(\left(1+|D y(x)|^{2}\right)^{\frac{\alpha-2}{2}}+\left|D\left(y_{\rho_{k}}-y\right)(x)\right|^{\alpha-2}\right)
\end{aligned}
$$


and by taking into account Lemma 4.7 , we deduce that

$$
\begin{aligned}
\left\|\left(1+\left|\sigma_{\rho_{k}}^{\varphi}\right|^{2}\right)^{\frac{\alpha-2}{4}} D z_{\rho_{k}}\right\|_{2}^{2} & =\int_{\Omega}\left(1+\left|\sigma_{\rho_{k}}^{\varphi}(x)\right|^{2}\right)^{\frac{\alpha-2}{2}}\left|D z_{\rho_{k}}(x)\right|^{2} \mathrm{~d} x \\
& \leq 2^{\alpha-2} \int_{\Omega}\left(\left(1+|D y(x)|^{2}\right)^{\frac{\alpha-2}{2}}+\left|D\left(y_{\rho_{k}}-y\right)(x)\right|^{\alpha-2}\right)\left|D z_{\rho_{k}}(x)\right|^{2} \mathrm{~d} x \\
& =2^{\alpha-2}\left(\left\|z_{\rho_{k}}\right\|_{H_{\alpha}^{y}}^{2}+\rho_{k}^{\alpha-2}\left\|D z_{\rho_{k}}\right\|_{\alpha}^{\alpha}\right) \\
& \leq\left(2^{2 \alpha+1}+\kappa_{3}^{2}\right)\left(\frac{\kappa_{2}}{\mu}\right)^{2}\left(\|v\|_{2}+\frac{\kappa_{1}}{\mu}\left(\|u\|_{2}+\|v\|_{2}\right)\|D f\|_{2}\right)^{2} .
\end{aligned}
$$

Therefore, the strong convergence of $\left(\sigma_{\rho_{k}}^{\varphi}\right)_{k}$ to $D y$ in $L^{\alpha}(\Omega)$ and the weak convergence of $\left(D z_{\rho_{k}}\right)_{k}$ to $D z$ in $L^{2}(\Omega)$ yield

$$
\left(1+\left|\sigma_{\rho_{k}}^{\varphi}\right|^{2}\right)^{\frac{\alpha-2}{4}} D z_{\rho_{k}} \longrightarrow\left(1+|D y|^{2}\right)^{\frac{\alpha-2}{4}} D z \quad \text { weakly in } L^{2}(\Omega) .
$$

On the other hand, let us consider the superposition (or Nemytskii) operator $\mathcal{A}$ acting from $\left(L^{\alpha}(\Omega)\right)^{n \times n}$ to $\left(L^{2}(\Omega)\right)^{n \times n}$ and defined by

$$
\mathcal{A}(g)(x)=\frac{D \varphi: \tau^{\prime}(g(x))}{\left(1+|g(x)|^{2}\right)^{\frac{\alpha-2}{4}}}, \quad x \in \Omega .
$$

Using $\mathbf{A} 1$, we can easily verify that

$$
|\mathcal{A}(g)|=\frac{\left|D \varphi: \tau^{\prime}(g)\right|}{\left(1+|g|^{2}\right)^{\frac{\alpha-2}{4}}} \leq \gamma n^{2} \frac{|D \varphi|\left(1+|g|^{2}\right)^{\frac{\alpha-2}{2}}}{\left(1+|g|^{2}\right)^{\frac{\alpha-2}{4}}}=\gamma n^{2}|D \varphi|\left(1+|g|^{2}\right)^{\frac{\alpha-2}{4}}
$$

which shows that $\mathcal{A}$ is continuous. Therefore, since $\left(\sigma_{\rho_{k}}^{\varphi}\right)_{k}$ converges to $D y$ in $L^{\alpha}(\Omega)$, we deduce that

$$
\mathcal{A}\left(\sigma_{\rho_{k}}^{\varphi}\right) \longrightarrow \mathcal{A}(D y) \quad \text { strongly in } L^{2}(\Omega) .
$$

Combining (4.12) and (4.13), we deduce that

$$
\begin{aligned}
\lim _{k \rightarrow+\infty}\left(\tau^{\prime}\left(\sigma_{\rho_{k}}^{\varphi}\right): D z_{\rho_{k}}, D \varphi\right) & =\lim _{k \rightarrow+\infty}\left(D \varphi: \tau^{\prime}\left(\sigma_{\rho_{k}}^{\varphi}\right), D z_{\rho_{k}}\right) \\
& =\lim _{k \rightarrow+\infty}\left(\mathcal{A}\left(\sigma_{\rho_{k}}^{\varphi}\right),\left(1+\left|\sigma_{\rho_{k}}^{\varphi}\right|^{2}\right)^{\frac{\alpha-2}{4}} D z_{\rho_{k}}\right) \\
& =\left(\mathcal{A}(D y),\left(1+|D y|^{2}\right)^{\frac{\alpha-2}{4}} D z\right) \\
& =\left(D \varphi: \tau^{\prime}(D y), D z\right)=\left(\tau^{\prime}(D y): D z, D \varphi\right)
\end{aligned}
$$

and the proof is complete.

Remark 4.10. Notice that following [18], we can avoid the use of the mean values theorem in (4.11) and obtain similar estimates and convergence results by adapting the proof. In particular, (4.12) may be replaced by

$$
\int_{0}^{1}\left(1+\left|D y+s D\left(y_{\rho_{k}}-y\right)\right|^{2}\right)^{\frac{\alpha-2}{4}} \mathrm{~d} s D z_{\rho_{k}} \longrightarrow\left(1+|D y|^{2}\right)^{\frac{\alpha-2}{4}} D z \quad \text { weakly in } L^{2}(\Omega)
$$

and the superposition operator $\mathcal{A}$ may be defined as

$$
\begin{gathered}
\mathcal{A}: L^{1}\left([0,1] ; \quad\left(L^{\alpha}(\Omega)\right)^{n \times n}\right) \longrightarrow\left(L^{2}(\Omega)\right)^{n \times n} \\
\mathcal{A}(g)(x)=\frac{D \varphi: \int_{0}^{1} \tau^{\prime}(g(s)(x)) \mathrm{d} s}{\left(\int_{0}^{1}\left(1+|g(s)(x)|^{2}\right)^{\frac{\alpha-2}{2}} \mathrm{~d} s\right)^{\frac{1}{2}}}, \quad x \in \Omega .
\end{gathered}
$$


Proposition 4.11. If $\left(z_{\rho_{k}}\right)_{k}$ weakly converges to $z$ in $H_{\alpha}^{y}$ for some sequence $\left(\rho_{k}\right)_{k}$ converging to zero, then $z$ is the unique solution of problem (4.8). Moreover, $\left(z_{\rho_{k}}\right)_{k}$ converges strongly to $z$ in $V_{2}$.

Proof. The first assertion is a direct consequence of Lemmas 4.8, 4.9 and of the density of $\mathcal{V}$ in $H_{\alpha}^{y}$. To prove the strong convergence, let us set

$$
M=\tau^{\prime}(D y(x)), \quad M_{\rho}(x)=\tau^{\prime}\left(\sigma_{\rho}(x)\right),
$$

where $\sigma_{\rho}$ is as in (4.11) with $\varphi$ substituted by $y_{\rho}-y$. Due to $\mathbf{A 2}$, the matrices

$$
M^{s}(x)=\frac{M(x)+M^{T}(x)}{2}, \quad M_{\rho}^{s}(x)=\frac{M_{\rho}(x)+M_{\rho}^{T}(x)}{2}
$$

are symmetric and positive definite. Applying the Cholesky method to $M^{s}(x)$ and $M_{\rho}^{s}(x)$, we deduce the existence of lower triangular matrices $L(x)$ and $L_{\rho}(x)$ such that

$$
M^{s}(x)=L(x) L^{T}(x) \quad \text { and } \quad M_{\rho}^{s}(x)=L_{\rho}(x) L_{\rho}^{T}(x) .
$$

Therefore, by taking into account (4.10) and (4.11), we obtain

$$
\begin{aligned}
\left\|L_{\rho_{k}}^{T} D z_{\rho_{k}}\right\|_{2}^{2} & =\left(M_{\rho_{k}}: D z_{\rho_{k}}, D z_{\rho_{k}}\right)=-\frac{1}{\rho_{k}}\left(w_{\rho_{k}} \cdot \nabla y_{\rho_{k}}-w \cdot \nabla y, z_{\rho_{k}}\right)+\left(v, z_{\rho_{k}}\right) \\
& =-\left(f \cdot \nabla y_{\rho_{k}}+y \cdot \nabla z_{\rho_{k}}, z_{\rho_{k}}\right)+\left(v, z_{\rho_{k}}\right)=-\left(f \cdot \nabla y_{\rho_{k}}, z_{\rho_{k}}\right)+\left(v, z_{\rho_{k}}\right) \\
& \leq \kappa_{1}\|D f\|_{2}\left\|D y_{\rho_{k}}\right\|_{2}\left\|D z_{\rho_{k}}\right\|_{2}+\kappa_{2}\|v\|_{2}\left\|D z_{\rho_{k}}\right\|_{2} \\
& \leq\left(\kappa_{2} \kappa_{1}\|D f\|_{2} \frac{\left\|u_{\rho_{k}}\right\|_{2}}{\mu}+\kappa_{2}\|v\|_{2}\right)\left\|D z_{\rho_{k}}\right\|_{2} \\
& \leq \kappa_{2}\left(\frac{\kappa_{1}}{\mu}\left(\|u\|_{2}+\|v\|_{2}\right)\|D f\|_{2}+\|v\|_{2}\right)\left\|D z_{\rho_{k}}\right\|_{2} \\
& \leq \frac{\kappa_{2}^{2}}{\mu}\left(\frac{\kappa_{1}}{\mu}\left(\|u\|_{2}+\|v\|_{2}\right)\|D f\|_{2}+\|v\|_{2}\right)^{2}
\end{aligned}
$$

and the sequence $\left(L_{\rho_{k}}^{T} D z_{\rho_{k}}\right)_{k}$ is bounded in $L^{2}(\Omega)$. Arguments similar to those used in the third step of the proof of Theorem 3.1 in [5] show that $\left(L_{\rho_{k}}\right)_{k}$ strongly converges to $L$ in $L^{2}(\Omega)$ and $\left(L_{\rho_{k}}^{T} D z_{\rho_{k}}\right)_{k}$ weakly converges to $L^{T} D z$ in $L^{2}(\Omega)$. Taking into account (4.14), we deduce that

$$
\begin{aligned}
\left\|L^{T} D z\right\|_{2}^{2} \leq \liminf _{k}\left\|L_{\rho_{k}}^{T} D z_{\rho_{k}}\right\|_{2}^{2} & \leq \limsup _{k}\left\|L_{\rho_{k}}^{T} D z_{\rho_{k}}\right\|_{2}^{2}=\underset{k}{\limsup }\left(M_{\rho_{k}}: D z_{\rho_{k}}, D z_{\rho_{k}}\right) \\
& =\underset{k}{\limsup }\left(-\frac{1}{\rho_{k}}\left(w_{\rho_{k}} \cdot \nabla y_{\rho_{k}}-w \cdot \nabla y, z_{\rho_{k}}\right)+\left(v, z_{\rho_{k}}\right)\right) \\
& =\limsup _{k}\left(-\left(f \cdot \nabla y_{\rho_{k}}+w \cdot \nabla z_{\rho_{k}}, z_{\rho_{k}}\right)+\left(v, z_{\rho_{k}}\right)\right) \\
& =\underset{k}{\limsup }\left(-\left(f \cdot \nabla y_{\rho_{k}}, z_{\rho_{k}}\right)+\left(v, z_{\rho_{k}}\right)\right)=-(f \cdot \nabla y, z)+(v, z) \\
& =-(f \cdot \nabla y-w \cdot \nabla z, z)+(v, z)=(M: D z, D z)=\left\|L^{T} D z\right\|_{2}^{2} .
\end{aligned}
$$

Weak convergence together with norm convergence implies strong convergence of $\left(L_{\rho_{k}}^{T} D z_{\rho_{k}}\right)_{k}$ to $L^{T} D z$ in $L^{2}(\Omega)$. The conclusion follows by following [5].

Proposition 4.12. Let $G: L^{2}(\Omega) \times V_{\alpha} \longrightarrow V_{2}$ be the functional defined by $G(w, u)=y$, where $y \equiv y_{w, u}$ is the solution of (4.1) corresponding to $(w, u)$. Then, $G$ is Gâteaux diferentiable at $(w, u)$ and its derivative in direction 
$(f, v), z=G^{\prime}(w, u)(f, v)$ is the unique solution in $H_{\alpha}^{y}$ of problem (4.8). Moreover, setting $I(w, u)=J\left(y_{w, u}, u\right)$ we have

$$
\frac{\partial I}{\partial w}(w, u) \cdot f=\left(y-y_{d}, z_{f}\right) \quad \text { and } \quad \frac{\partial I}{\partial u}(w, u) \cdot v=\left(y, \phi_{v}\right)+\nu(u, v),
$$

where $z_{f} \in H_{\alpha}^{y}$ is the solution of (4.8) corresponding to $v=0$ and $\phi_{v} \in H_{\alpha}^{y}$ is the solution of (4.8) corresponding to $f=0$.

Proof. For $\rho \in] 0,1\left[\right.$, let $w_{\rho}=w+\rho f, u_{\rho}=u+\rho v, y_{\rho} \equiv y_{w_{\rho}, u_{\rho}}$ and $z_{\rho}=\frac{y_{\rho}-y}{\rho}$. Due to Lemma 4.7, we deduce that $\left(z_{\rho}\right)_{\rho}$ is bounded in $H_{\alpha}^{y}$. There then exist a subsequence $\left(z_{\rho_{k}}\right)_{k}$ and $z \in H_{\alpha}^{y}$ such that $\left(z_{\rho_{k}}\right)_{k}$ weakly converges to $z$ in $V_{2}$. Due to Proposition 4.11, $z$ is the solution of the linear equation (4.8) and $\left(z_{\rho_{k}}\right)_{k}$ strongly converges to $z$ in $V_{2}$. Taking into account Lemma 4.7, we deduce that

$$
\begin{aligned}
\|D z\|_{2}=\lim _{k}\left\|D z_{\rho_{k}}\right\|_{2} & \leq \frac{\kappa_{2}}{\mu}\left(\|v\|_{2}+\frac{\kappa_{1}\|u\|_{2}}{\mu}\|D f\|_{2}\right) \\
& \leq \frac{\kappa_{2}}{\mu}\left(\|v\|_{2}+\frac{\kappa_{1}\|u\|_{2}|\Omega|^{\frac{\alpha-2}{\alpha}}}{\mu}\|D f\|_{\alpha}\right) \leq \frac{\kappa_{2}}{\mu}\left(1+\frac{\kappa_{1}\|u\|_{2}|\Omega|^{\frac{\alpha-2}{\alpha}}}{\mu}\right)\left(\|v\|_{2}+\|D f\|_{\alpha}\right)
\end{aligned}
$$

which implies the continuity of $G^{\prime}(w, u): L^{2}(\Omega) \times V_{\alpha} \rightarrow V_{2}$ and therefore the Gâteaux differentiability of $G$. The last part is a consequence of the differentiability of $J$.

\section{Approximate optimality conditions}

The following result states the optimality conditions for $\left(P_{\alpha}^{\varepsilon}\right)$.

Theorem 5.1. Let $\left(w^{\varepsilon}, u^{\varepsilon}\right)$ be a solution of $\left(P_{\alpha}^{\varepsilon}\right)$ and let $y^{\varepsilon} \in V_{\alpha}$ be the corresponding solution of (4.1). Then there exists $p^{\varepsilon} \in H_{\alpha}^{y^{\varepsilon}}$ such that

$$
\begin{gathered}
\left(\tau\left(D y^{\varepsilon}\right), D \varphi\right)+\left(w^{\varepsilon} \cdot \nabla y^{\varepsilon}, \varphi\right)=\left(u^{\varepsilon}, \varphi\right) \quad \text { for all } \varphi \in V_{\alpha}, \\
\left(\tau^{\prime}\left(D y^{\varepsilon}\right): D \varphi, D p^{\varepsilon}\right)-\left(w^{\varepsilon} \cdot \nabla p^{\varepsilon}, \varphi\right)-\left(\varphi \cdot \nabla p^{\varepsilon}, y^{\varepsilon}\right)=\left(2 y^{\varepsilon}-\bar{y}-y_{d}, \varphi\right) \quad \text { for all } \varphi \in H_{\alpha}^{y^{\varepsilon}}
\end{gathered}
$$

and

$$
\left(p^{\varepsilon}+\nu u^{\varepsilon}+u^{\varepsilon}-\bar{u}, v-u^{\varepsilon}\right) \geq 0 \quad \text { for all } v \in U_{a d} .
$$

Proof. Let $p^{\varepsilon} \in H_{\alpha}^{y^{\varepsilon}}$ be the solution of

$$
\left(\tau^{\prime}\left(D y^{\varepsilon}\right): D \varphi, D p^{\varepsilon}\right)-\left(w^{\varepsilon} \cdot \nabla p^{\varepsilon}, \varphi\right)=\left(2 y^{\varepsilon}-\bar{y}-y_{d}, \varphi\right)+\frac{1}{\varepsilon}\left(D\left(y^{\varepsilon}-w^{\varepsilon}\right), D \varphi\right) \quad \text { for all } \varphi \in H_{\alpha}^{y^{\varepsilon}} .
$$

(Existence and uniqueness of $p^{\varepsilon}$ can be obtained with arguments similar to those used in the proof of Prop. 4.6.) Since $J^{\varepsilon}$ is differentiable, by taking into account Proposition 4.12, we obtain

$$
\frac{\partial J^{\varepsilon}}{\partial w}\left(w^{\varepsilon}, u^{\varepsilon}\right) \cdot f=\left(2 y^{\varepsilon}-\bar{y}-y_{d}, z_{f}^{\varepsilon}\right)+\frac{1}{\varepsilon}\left(D\left(y^{\varepsilon}-w^{\varepsilon}\right), D\left(z_{f}^{\varepsilon}-f\right)\right),
$$

where $z_{f}^{\varepsilon} \in H_{\alpha}^{y^{\varepsilon}}$ is the solution of (4.8) corresponding to $(y, w, v)=\left(y^{\varepsilon}, w^{\varepsilon}, 0\right)$, and

$$
\frac{\partial J^{\varepsilon}}{\partial u}\left(w^{\varepsilon}, u^{\varepsilon}\right) \cdot v=\left(2 y^{\varepsilon}-\bar{y}-y_{d}, \phi_{v}^{\varepsilon}\right)+\frac{1}{\varepsilon}\left(D\left(y^{\varepsilon}-w^{\varepsilon}\right), D \phi_{v}^{\varepsilon}\right)+\left(\nu u^{\varepsilon}+u^{\varepsilon}-\bar{u}, v\right),
$$


where $\phi_{v}^{\varepsilon} \in H_{\alpha}^{y^{\varepsilon}}$ is the solution of (4.8) corresponding to $(y, w, f)=\left(y^{\varepsilon}, w^{\varepsilon}, 0\right)$. Setting $\varphi=z_{f}^{\varepsilon}$ in (5.4), by taking into account (5.5) and the weak formulation of (4.8), it follows that

$$
\begin{aligned}
\frac{\partial J^{\varepsilon}}{\partial w}\left(w^{\varepsilon}, u^{\varepsilon}\right) \cdot f & =\left(\tau^{\prime}\left(D y^{\varepsilon}\right): D z_{f}^{\varepsilon}, D p^{\varepsilon}\right)-\left(w^{\varepsilon} \cdot \nabla p^{\varepsilon}, z_{f}^{\varepsilon}\right)-\frac{1}{\varepsilon}\left(D\left(y^{\varepsilon}-w^{\varepsilon}\right), f\right) \\
& =\left(\tau^{\prime}\left(D y^{\varepsilon}\right): D z_{f}^{\varepsilon}, D p^{\varepsilon}\right)+\left(w^{\varepsilon} \cdot \nabla z_{f}^{\varepsilon}, p^{\varepsilon}\right)-\frac{1}{\varepsilon}\left(D\left(y^{\varepsilon}-w^{\varepsilon}\right), f\right) \\
& =-\left(f \cdot \nabla y^{\varepsilon}, p^{\varepsilon}\right)-\frac{1}{\varepsilon}\left(D\left(y^{\varepsilon}-w^{\varepsilon}\right), f\right) \\
& =\left(f \cdot \nabla p^{\varepsilon}, y^{\varepsilon}\right)-\frac{1}{\varepsilon}\left(D\left(y^{\varepsilon}-w^{\varepsilon}\right), f\right) .
\end{aligned}
$$

Similarly, by setting $\varphi=\phi_{v}^{\varepsilon}$ in (5.4) and taking into account (5.6) and the weak formulation of (4.8), it follows that

$$
\begin{aligned}
\frac{\partial J^{\varepsilon}}{\partial u}\left(w^{\varepsilon}, u^{\varepsilon}\right) \cdot v & =\left(\tau^{\prime}\left(D y^{\varepsilon}\right): D \phi_{v}^{\varepsilon}, D p^{\varepsilon}\right)-\left(w^{\varepsilon} \cdot \nabla p^{\varepsilon}, \phi_{v}^{\varepsilon}\right)+\left(\nu u^{\varepsilon}+u^{\varepsilon}-\bar{u}, v\right) \\
& =\left(\tau^{\prime}\left(D y^{\varepsilon}\right): D \phi_{v}^{\varepsilon}, D p^{\varepsilon}\right)+\left(w^{\varepsilon} \cdot \nabla \phi_{v}^{\varepsilon}, p^{\varepsilon}\right)+\left(\nu u^{\varepsilon}+u^{\varepsilon}-\bar{u}, v\right) \\
& =\left(v, p^{\varepsilon}\right)+\left(\nu u^{\varepsilon}+u^{\varepsilon}-\bar{u}, v\right) \\
& =\left(p^{\varepsilon}+\nu u^{\varepsilon}+u^{\varepsilon}-\bar{u}, v\right) .
\end{aligned}
$$

On the other hand, since $\left(w^{\varepsilon}, u^{\varepsilon}\right)$ is a solution of $\left(P_{\alpha}^{\varepsilon}\right)$, we have

$$
\begin{cases}\frac{\partial J^{\varepsilon}}{\partial w}\left(w^{\varepsilon}, u^{\varepsilon}\right) \cdot f=0 & \text { for all } f \in V_{\alpha} \\ \frac{\partial J^{\varepsilon}}{\partial u}\left(w^{\varepsilon}, u^{\varepsilon}\right) \cdot\left(v-u^{\varepsilon}\right) \geq 0 & \text { for all } v \in U_{a d} .\end{cases}
$$

These relations together with (5.7) and (5.8) imply the optimality condition (5.3) and

$$
\left(f \cdot \nabla p^{\varepsilon}, y^{\varepsilon}\right)=\frac{1}{\varepsilon}\left(D\left(y^{\varepsilon}-w^{\varepsilon}\right), f\right) \quad \text { for all } f \in V_{\alpha} .
$$

Relation (5.2) is then a direct consequence of (5.4) and (5.9).

\section{Proof of the MAIN RESUlt}

The proof is split into four steps.

Step 1. Estimate for $p^{\varepsilon}$.

Setting $\varphi=p^{\varepsilon}$ in (5.2), and using assumption A2 and Lemma 2.4 we obtain

$$
\begin{aligned}
\mu\left\|p^{\varepsilon}\right\|_{H_{\alpha}^{y^{\varepsilon}}}^{2} & \leq\left(\tau^{\prime}\left(D y^{\varepsilon}\right): D p^{\varepsilon}, D p^{\varepsilon}\right) \\
& =\left(w^{\varepsilon} \cdot \nabla p^{\varepsilon}, p^{\varepsilon}\right)+\left(p^{\varepsilon} \cdot \nabla p^{\varepsilon}, y^{\varepsilon}\right)+\left(y^{\varepsilon}-y_{d}, p^{\varepsilon}\right)+\left(y^{\varepsilon}-\bar{y}, p^{\varepsilon}\right) \\
& =-\left(p^{\varepsilon} \cdot \nabla y^{\varepsilon}, p^{\varepsilon}\right)+\left(y^{\varepsilon}-y_{d}, p^{\varepsilon}\right)+\left(y^{\varepsilon}-\bar{y}, p^{\varepsilon}\right) .
\end{aligned}
$$

Using classical arguments, we have the following estimate

$$
\begin{aligned}
\left|-\left(p^{\varepsilon} \cdot \nabla y^{\varepsilon}, p^{\varepsilon}\right)+\left(y^{\varepsilon}-y_{d}, p^{\varepsilon}\right)+\left(y^{\varepsilon}-\bar{y}, p^{\varepsilon}\right)\right| & \leq\left\|\nabla y^{\varepsilon}\right\|_{2}\left\|p^{\varepsilon}\right\|_{4}^{2}+\left(\left\|y^{\varepsilon}-y_{d}\right\|_{2}+\left\|y^{\varepsilon}-\bar{y}\right\|_{2}\right)\left\|p^{\varepsilon}\right\|_{2} \\
& \leq\left\|\nabla y^{\varepsilon}\right\|_{2}\left\|p^{\varepsilon}\right\|_{4}^{2}+\frac{1}{2}\left\|y^{\varepsilon}-y_{d}\right\|_{2}^{2}+\frac{1}{2}\left\|y^{\varepsilon}-\bar{y}\right\|_{2}^{2}+\left\|p^{\varepsilon}\right\|_{2}^{2} \\
& \leq\left\|\nabla y^{\varepsilon}\right\|_{2}\left\|p^{\varepsilon}\right\|_{4}^{2}+J^{\varepsilon}\left(w^{\varepsilon}, u^{\varepsilon}\right)+\left\|p^{\varepsilon}\right\|_{2}^{2} \\
& \leq\left\|\nabla y^{\varepsilon}\right\|_{2}\left\|p^{\varepsilon}\right\|_{4}^{2}+J(\bar{y}, \bar{u})+\left\|p^{\varepsilon}\right\|_{2}^{2} .
\end{aligned}
$$


Moreover, recalling the inequality (see [8], Chap. 2)

$$
\left\|p^{\varepsilon}\right\|_{4} \leq \kappa_{4}\left\|p^{\varepsilon}\right\|_{2}^{1-\frac{n}{4}}\left\|\nabla p^{\varepsilon}\right\|_{2}^{\frac{n}{4}} \quad \text { with } \kappa_{4}=\left(\frac{2(n-1)}{\sqrt{n^{3}}}\right)^{\frac{n}{4}},
$$

by using the Korn inequality, Proposition 4.1 and estimate (4.3), we deduce that

$$
\begin{aligned}
\left\|\nabla y^{\varepsilon}\right\|_{2}\left\|p^{\varepsilon}\right\|_{4}^{2} & \leq \kappa_{4}^{2}\left\|\nabla y^{\varepsilon}\right\|_{2}\left\|p^{\varepsilon}\right\|_{2}^{2-\frac{n}{2}}\left\|\nabla p^{\varepsilon}\right\|_{2}^{\frac{n}{2}} \\
& \leq 2^{\frac{n+2}{4}} \kappa_{4}^{2}\left\|D y^{\varepsilon}\right\|_{2}\left\|p^{\varepsilon}\right\|_{2}^{2-\frac{n}{2}}\left\|D p^{\varepsilon}\right\|_{2}^{\frac{n}{2}} \\
& \leq 2^{\frac{n+2}{4}} \kappa_{4}^{2}\left\|D y^{\varepsilon}\right\|_{2}\left\|p^{\varepsilon}\right\|_{2}^{2-\frac{n}{2}}\left\|p^{\varepsilon}\right\|_{H_{\alpha}^{\frac{n}{2}}}^{\frac{y^{\varepsilon}}{2}} \\
& \leq 2^{\frac{n}{4}+1} \frac{\kappa_{2} \kappa_{4}^{2}}{\mu}\left(2 J(\bar{y}, \bar{u})+\|\bar{u}\|_{2}\right)^{\frac{1}{2}}\left\|p^{\varepsilon}\right\|_{2}^{2-\frac{n}{2}}\left\|p^{\varepsilon}\right\|_{H_{\alpha}^{y^{\varepsilon}}}^{\frac{n}{2}} .
\end{aligned}
$$

Due to the Young inequality, for all $\theta>0$ we have

$2^{\frac{n}{4}+1} \frac{\kappa_{2} \kappa_{4}^{2}}{\mu}\left(2 J(\bar{y}, \bar{u})+\|\bar{u}\|_{2}\right)^{\frac{1}{2}}\left\|p^{\varepsilon}\right\|_{2}^{2-\frac{n}{2}}\left\|p^{\varepsilon}\right\|_{H_{\alpha}^{y^{\varepsilon}}}^{\frac{n}{2}} \leq \frac{4-n}{4 \theta^{\frac{4}{4-n}}}\left(2^{\frac{n}{4}+1} \frac{\kappa_{2} \kappa_{4}^{2}}{\mu}\left(2 J(\bar{y}, \bar{u})+\|\bar{u}\|_{2}\right)^{\frac{1}{2}}\right)^{\frac{4}{4-n}}\left\|p^{\varepsilon}\right\|_{2}^{2}+\frac{n \theta^{\frac{4}{n}}}{4}\left\|p^{\varepsilon}\right\|_{H_{\alpha}^{y^{\varepsilon}}}^{2}$.

Choosing $\theta=\mu^{\frac{n}{4}}$, using assumption A2 and combining (6.1)-(6.3), we deduce that

$$
\left\|p^{\varepsilon}\right\|_{H_{\alpha}^{y^{\varepsilon}}}^{2} \leq\left(\left(2^{\frac{n}{4}+1} \frac{\kappa_{2} \kappa_{4}^{2}}{\mu^{2}}\left(2(\bar{u})+\|\bar{u}\|_{2}\right)^{\frac{1}{2}}\right)^{\frac{4}{4-n}}+\frac{n}{(4-n) \mu}\right)\left\|p^{\varepsilon}\right\|_{2}^{2}+\frac{n J(\bar{y}, \bar{u})}{(4-n) \mu} .
$$

Step 2. Passage to the limit when $p^{\varepsilon}$ is bounded in $L^{2}(\Omega)$.

Let us assume that $\left(p^{\varepsilon}\right)_{\varepsilon}$ is bounded in $L^{2}(\Omega)$. Due to (6.4), it follows that $\left(p^{\varepsilon}\right)_{\varepsilon}$ is bounded in $H_{\alpha}^{y^{\varepsilon}}$ (and thus in $V_{2}$ ). There then exist a subsequence $\left(p^{\varepsilon_{k}}\right)_{k}$ and $\bar{p} \in V_{2}$ such that $\left(p^{\varepsilon_{k}}\right)_{k}$ weakly converges to $\bar{p}$ in $V_{2}$. Arguing as in the proof of Lemma 4.9 and taking into account Proposition 4.2, we deduce that

$$
\lim _{k \rightarrow+\infty}\left(\tau^{\prime}\left(D y^{\varepsilon_{k}}\right): D \varphi, D p_{\varepsilon_{k}}\right)=\left(\tau^{\prime}(D \bar{y}): D \varphi, D \bar{p}\right) \quad \text { for all } \varphi \in \mathcal{V}
$$

and

$$
\lim _{k \rightarrow+\infty}\left(\left(w^{\varepsilon_{k}} \cdot \nabla p^{\varepsilon_{k}}, \varphi\right)+\left(\varphi \cdot \nabla p^{\varepsilon_{k}}, y^{\varepsilon}\right)\right)=(\bar{y} \cdot \nabla \bar{p}, \varphi)+(\varphi \cdot \nabla \bar{p}, \bar{y})
$$

for all $\varphi \in V_{2}$. Therefore, by passing to the limit in the weak formulation satisfied to $y^{\varepsilon_{k}}$, we deduce that

$$
\left(\tau^{\prime}(D \bar{y}): D \varphi, D \bar{p}\right)-(\bar{y} \cdot \nabla \bar{p}, \varphi)-(\varphi \cdot \nabla \bar{p}, \bar{y})=\left(\bar{y}-y_{d}, \varphi\right)
$$

for all $\varphi \in \mathcal{V}$.

Step 3. $\bar{p}$ belongs to $V_{\alpha}^{\bar{y}}$.

Let us now prove that $\left(1+|D \bar{y}|^{2}\right)^{\frac{\alpha-2}{2}} D \bar{p}$ belongs to $L^{2}(\Omega)$. Set

$$
M=\tau^{\prime}(D \bar{y}(x)), \quad M^{\varepsilon}(x)=\tau^{\prime}\left(D y^{\varepsilon}(x)\right),
$$

and

$$
M^{s}(x)=\frac{M(x)+M^{T}(x)}{2}, \quad M^{\varepsilon, s}(x)=\frac{M^{\varepsilon}(x)+\left(M^{\varepsilon}(x)\right)^{T}}{2}
$$


Due to A2, the matrices $M^{s}(x)$ and $M^{\varepsilon, s}(x)$ are symmetric and positive definite. Applying the Cholesky method to $M^{s}(x)$ and $M^{\varepsilon, s}(x)$, we deduce the existence of lower triangular matrices $L(x)$ and $L^{\varepsilon}(x)$ such that

$$
M^{s}(x)=L(x) L^{T}(x) \quad \text { and } \quad M^{\varepsilon, s}(x)=L^{\varepsilon}(x)\left(L^{\varepsilon}(x)\right)^{T} .
$$

Therefore, taking into account (6.1) and (6.2), we have

$$
\begin{aligned}
\left\|\left(L^{\varepsilon}\right)^{T} D p^{\varepsilon}\right\|_{2}^{2} & =\left(M^{\varepsilon}: D p^{\varepsilon}, D p^{\varepsilon}\right)=-\left(p^{\varepsilon} \cdot \nabla y^{\varepsilon}, p^{\varepsilon}\right)+\left(y^{\varepsilon}-y_{d}, p^{\varepsilon}\right)+\left(y^{\varepsilon}-\bar{y}, p^{\varepsilon}\right) \\
& \leq\left\|\nabla y^{\varepsilon}\right\|_{2}\left\|p^{\varepsilon}\right\|_{4}^{2}+J(\bar{y}, \bar{u})+\left\|p^{\varepsilon}\right\|_{2}^{2}
\end{aligned}
$$

and the sequence $\left(\left(L^{\varepsilon}\right)^{T} D p^{\varepsilon}\right)_{\varepsilon}$ is bounded in $L^{2}(\Omega)$. On the other hand, due to $\mathbf{A} 1$ we have

$$
\left|L^{\varepsilon}(x)\right|^{2}=\left|M^{\varepsilon}(x)\right| \leq \gamma n^{2}\left(1+\left|D y^{\varepsilon}(x)\right|^{2}\right)^{\frac{\alpha-2}{2}} \in L^{\frac{\alpha}{\alpha-2}}(\Omega)
$$

for all $x \in \Omega$. The convergence of $\left(D y^{\varepsilon}\right)_{\varepsilon}$ to $D \bar{y}$ and the weak convergence of $\left(p^{\varepsilon}\right)_{\varepsilon}$ to $\bar{p}$ in $V_{2}$ together with arguments similar to those used in the proof of Proposition 4.11 show that

$$
\left(L^{\varepsilon}\right)^{T} D p^{\varepsilon} \longrightarrow L^{T} D \bar{p} \quad \text { weakly in } L^{2}(\Omega) .
$$

Moreover, we have

$$
\begin{aligned}
\mu\left\|\left(1+|D \bar{y}|^{2}\right)^{\frac{\alpha-2}{4}} D \bar{p}\right\|_{2}^{2} & \leq(M: D \bar{p}, D \bar{p})=\left\|L^{T} D \bar{p}\right\|_{2}^{2} \\
& \leq \liminf _{\varepsilon}\left\|\left(L^{\varepsilon}\right)^{T} D p^{\varepsilon}\right\|_{2}^{2} \leq \limsup _{\varepsilon}\left\|\left(L^{\varepsilon}\right)^{T} D p^{\varepsilon}\right\|_{2}^{2}=\underset{\varepsilon}{\limsup }\left(M^{\varepsilon}: D p^{\varepsilon}, D p^{\varepsilon}\right) \\
& =\limsup _{\varepsilon}\left(-\left(p^{\varepsilon} \cdot \nabla y^{\varepsilon}, p^{\varepsilon}\right)+\left(y^{\varepsilon}-y_{d}, p^{\varepsilon}\right)+\left(y^{\varepsilon}-\bar{y}, p^{\varepsilon}\right)\right) \\
& =-(\bar{p} \cdot \nabla \bar{y}, \bar{p})+\left(\bar{y}-y_{d}, \bar{p}\right)
\end{aligned}
$$

which implies (3.5). This inequality, together with (6.5) and the density of $\mathcal{V}$ in $H_{\alpha}^{\bar{y}}$ give (3.3) with $\bar{\lambda}=1$. Similarly, we can pass to the limit in (5.1) and (5.3) and derive (3.2) and (3.4). Notice here that we can guarantee that the sequence $\left(p^{\varepsilon}\right)_{\varepsilon}$ is compact in $H_{\alpha}^{y^{\varepsilon}}$ and that its limit point belongs to $V_{\alpha}^{\bar{y}}$. However, there is no reason to assume that the sequence converges to an element of $H_{\alpha}^{\bar{y}}$. This is closely related to the fact that the space $\mathcal{V}$ is not dense in $V_{\alpha}^{\bar{y}}$ and to the nonuniqueness of the limit problem.

Step 4. Passage to the limit when $p^{\varepsilon}$ is not bounded in $L^{2}(\Omega)$.

If $\left(p^{\varepsilon}\right)$ is not bounded in $L^{2}(\Omega)$, we set

$$
\lambda^{\varepsilon}=\frac{1}{\left\|p^{\varepsilon}\right\|_{2}} \longrightarrow 0 \quad \text { when } \varepsilon \rightarrow 0
$$

and denote again $\lambda^{\varepsilon} p^{\varepsilon}$ by $p^{\varepsilon}$. Using optimality conditions (5.2) and (5.3), we obtain

$$
\left(\tau^{\prime}\left(D y^{\varepsilon}\right): D \varphi, D p^{\varepsilon}\right)-\left(w^{\varepsilon} \cdot \nabla p^{\varepsilon}, \varphi\right)-\left(\varphi \cdot \nabla p^{\varepsilon}, y^{\varepsilon}\right)=\lambda^{\varepsilon}\left(y^{\varepsilon}-y_{d}, \varphi\right)+\lambda^{\varepsilon}\left(y^{\varepsilon}-\bar{y}, \varphi\right) \quad \text { for all } \varphi \in H_{\alpha}^{y^{\varepsilon}},
$$

and

$$
\left(p^{\varepsilon}+\lambda^{\varepsilon}\left(\nu u^{\varepsilon}+u^{\varepsilon}-\bar{u}\right), v-u^{\varepsilon}\right) \geq 0 \quad \text { for all } v \in U_{a d}
$$

respectively. Repeating the arguments stated in step 1-step 3, we can derive the optimality conditions (3.2)(3.4) with $\bar{\lambda}=0$. Finally, to prove (3.1) in this case, observe that since $\left(p^{\varepsilon}\right)$ weakly converges to $\bar{p}$ in $V_{2}$, it converges strongly in $L^{2}(\Omega)$. Therefore,

$$
\|\bar{p}\|_{2}=\lim _{\varepsilon}\left\|p^{\varepsilon}\right\|_{2}=1
$$

and thus $\bar{p} \neq 0$. 


\section{REFERENCES}

[1] F. Abergel and E. Casas, Some optimal control problems of multistate equations appearing in fluid mechanics. RAIRO Modél. Math. Anal. Numér. 27 (1993) 223-247.

[2] N. Arada, Optimal Control of shear-thickening flows. Departamento de Matemática, FCT-UNL, Portugal, Technical Report 3 (2012).

[3] E. Casas, Boundary control problems for quasi-linear elliptic equations: a Pontryagin's principle. Appl. Math. Optim. 33 (1996) 265-291.

[4] E. Casas and L.A. Fernández, Boundary control of quasilinear elliptic equations. INRIA, Rapport de Recherche 782 (1988).

[5] E. Casas and L.A. Fernández, Distributed control of systems governed by a general class of quasilinear elliptic equations. J. Differ. Equ. 35 (1993) 20-47.

[6] J.C. De Los Reyes and R. Griesse, State-constrained optimal control of the three-dimensional stationary Navier-Stokes equations. J. Math. Anal. Appl. 343 (2008) 257-272.

[7] J. Frehse, J. Málek and M. Steinhauer, An existence result for fluids with shear dependent viscosity-steady flows. Nonlinear. Anal. 30 (1997) 3041-3049.

[8] G.P. Galdi, An introduction to the mathematical theory of the Navier-Stokes equations, I, II, 2nd edition. Springer-Verlag, New York. Springer Tracts in Natural Philosophy 38, 39 (1998).

[9] M.D. Gunzburger and C. Trenchea, Analysis of an optimal control problem for the three-dimensional coupled modified NavierStokes and maxwell equations. J. Math. Anal. Appl. 333 (2007) 295-310.

[10] M.D. Gunzburger, L. Hou and T.P. Svobodny, Boundary velocity control of incompressible flow with an application to viscous drag reduction. SIAM J. Control Optim. 30 (1992) 167-181.

[11] C.O. Horgan, Korn's inequalities and their applications in continuum mechanics. SIAM Rev. 37 (1995) $491-511$.

[12] O.A. Ladyzhenskaya, The mathematical theory of viscous incompressible flow. Gordon and Beach, New York (1969).

[13] J.-L. Lions, Quelques méthodes de résolution des problèmes aux limites non linéaires. Dunod, Gauthier-Villars, Paris (1969).

[14] P. Kaplický, J. Málek and J. Stará, $C^{1, \alpha}$-solutions to a class of nonlinear fluids in two dimensions-stationary Dirichlet problem. Zap. Nauchn. Sem. POMI 259 (1999) 89-121.

[15] K. Kunisch and X. Marduel, Optimal control of non-isothermal viscoelastic fluid flow. J. Non-Newton. Fluid Mech. 88 (2000) 261-301.

[16] J. Nečas, J. Málek, J. Rokyta and M. Ružička, Weak and measure-valued solutions to evolutionary partial differential equations, Chapmann and Hall, London. Appl. Math. Math. Comput. 13 (1996).

[17] T. Roubíček and F. Tröltzsch, Lipschitz stability of optimal controls for the steady-state Navier-Stokes equations. Control Cybernet. 32 (2003) 683-705.

[18] T. Slawig, Distributed control for a class of non-Newtonian fluids. J. Differ. Equ. 219 (2005) 116-143.

[19] D. Wachsmuth and T. Roubíček, Optimal control of incompressible non-Newtonian fluids. Z. Anal. Anwend. 29 (2010) 351-376. 